\title{
TRANSITIONAL DISKS AS SIGNPOSTS OF YOUNG, MULTIPLANET SYSTEMS
}

\author{
Sarah E. Dodson-Robinson and Colette Salyk \\ Astronomy Department, University of Texas at Austin, 1 University Station C1400, Austin, TX 78712, USA; sdr@astro.as.utexas.edu \\ Received 2011 April 7; accepted 2011 June 23; published 2011 August 18
}

\begin{abstract}
Although there has yet been no undisputed discovery of a still-forming planet embedded in a gaseous protoplanetary disk, the cleared inner holes of transitional disks may be signposts of young planets. Here, we show that the subset of accreting transitional disks with wide, optically thin inner holes of $15 \mathrm{AU}$ or more can only be sculpted by multiple planets orbiting inside each hole. Multiplanet systems provide two key ingredients for explaining the origins of transitional disks. First, multiple planets can clear wide inner holes where single planets open only narrow gaps. Second, the confined, non-axisymmetric accretion flows produced by multiple planets provide a way for an arbitrary amount of mass transfer to occur through an apparently optically thin hole without overproducing infrared excess flux. Rather than assuming that the gas and dust in the hole are evenly and axisymmetrically distributed, one can construct an inner hole with apparently optically thin infrared fluxes by covering a macroscopic fraction of the hole's surface area with locally optically thick tidal tails. We also establish that other clearing mechanisms, such as photoevaporation, cannot explain our subset of accreting transitional disks with wide holes. Transitional disks are therefore high-value targets for observational searches for young planetary systems.
\end{abstract}

Key words: accretion, accretion disks - hydrodynamics - infrared: planetary systems - planet-disk interactions planets and satellites: formation - protoplanetary disks - stars: pre-main sequence

Online-only material: color figures

\section{INTRODUCTION}

Protoplanetary disks of gas and dust surrounding young stars are the birthplaces of planets-yet to date, there is still no undisputed observation of a still-forming planet in a gaseous disk. Astronomers have therefore paid considerable attention to transitional disks - protoplanetary disks with inner clearings - because such clearings occur as the result of tidal interactions between disks and planets (e.g., Lin \& Papaloizou 1993). To date, over 30 transitional disks have been discovered with the infrared spectrograph (IRS) instrument aboard the Spitzer Space Telescope (Muzerolle et al. 2010). If theorists were to demonstrate a planetary origin for any inner hole in a transitional disk, that disk would harbor extraordinary discovery potential for young, still-forming planets. Indeed, gaps in debris disks surrounding main-sequence stars harbor the giant planets Fomalhaut b (Kalas et al. 2008), $\epsilon$ Eridani b (Hatzes et al. 2000), the HR 8799 system (Marois et al. 2008, 2010), and $\beta$ Pictoris b (Lagrange et al. 2010).

Inner holes in gaseous protoplanetary disks with ages $\sim 1-10 \mathrm{Myr}$ are initially identified by the flux deficits they leave in the near-IR and/or mid-IR part of the disk's spectral energy distribution (SED). The observed flux deficits correspond to "missing" dust with characteristic temperatures of 200-1000 K (e.g., Calvet et al. 2002; Furlan et al. 2006; Brown et al. 2007). Model SEDs of the first transitional disks to be discovered, GM Aur (Koerner et al. 1993; Calvet et al. 2005) and TW Hya (Calvet et al. 2002), were based on disks with small inner holes only 3-4 AU wide. Soon after, investigations of planet-disk interactions demonstrated that such clearings could naturally be produced by a single Jupiter-mass planet (Quillen et al. 2004; Rice et al. 2006). Although the planet opened only a narrow gap, not a hole extending all the way to the central star, the dynamical studies showed that the residual gas and dust inside the gap would accrete onto the central star on the viscous timescale, widening the initial gap into a small, optically thin hole.

The first hole-size measurements performed with millimeter interferometers showed remarkable consistency with SEDbased models (Brown et al. 2009). However, further spatially resolved imaging of transitional disks brought a surprise. Some disks for which small, 3-4 AU inner holes were inferred based on SED modeling-including the canonical transitional disk, GM Aur-were shown to have large inner clearings of $15 \mathrm{AU}$ or more once imaged at high angular resolution (Hughes et al. 2009; Andrews et al. 2010, 2011). Yet, as the models of Rice et al. (2006) and Quillen et al. (2004) hinted, each single planet can open only a narrow gap, which must then widen into a hole by viscous clearing of the material inside the gap. The extraordinarily long viscous timescales at distances of $15 \mathrm{AU}$ or more meant the simple theory that each transitional disk hole contained a single giant planet was no longer satisfactory.

The prosaic explanation for wide holes in transitional disks - tidal truncation of the disk by a stellar companion, as observed in CoKu Tau/4 (Ireland \& Kraus 2008) - is also not likely responsible for the unusual and complex disks that concern us in this article. Based on observations with the Keck interferometer, Pott et al. (2010) rule out stellar companions in 0.35-4 AU orbits at the $99.7 \%$ confidence level in the holes of DM Tau, GM Aur, LkCa 15, UX Tau A, and RY Tau—at least three of which are examples of transitional disks with wide holes (Andrews et al. 2011). Kraus et al. (2011) also found no stellar companions in the inner holes of DM Tau, GM Aur, LkCa 15, and UX Tau A in their non-redundant aperture-masking survey of the Taurus star-forming region. The high accretion rates in these sources (see Table 1, and references therein), as well as the presence of inner disk dust and gas (e.g., Salyk et al. 2009; Espaillat et al. 2010), are also consistent with sub-stellar rather than stellar companions, as they indicate that disk truncation is incomplete. The set of transitional disks with large inner holes, 
Table 1

Observed Hole Sizes and Accretion Rates of Transitional Disks

\begin{tabular}{lcccr}
\hline \hline Name & $\begin{array}{c}\text { Hole Size } \\
(\text { AU })\end{array}$ & Ref & $\begin{array}{c}\dot{M} \\
\left(\log \left(M_{\odot} \mathrm{yr}^{-1}\right)\right)\end{array}$ & Ref \\
\hline DM Tau & 19 & 1 & -8.7 & 11 \\
DoAr 44 & 30 & 1 & -8.0 & 4 \\
GM Aur & 20 & 7 & -8.0 & 8 \\
HD 135344 & 39 & 3 & -8.3 & 6 \\
Lk H $\alpha$ 330 & 68,47 & 1,3 & -8.8 & $5^{\mathrm{a}}$ \\
MWC 758 & 73 & 1 & -8.0 & 1 \\
SR 24 S & 29 & 1 & -7.2 & 9 \\
T Cha & 15 & 2 & -8.4 & 10 \\
UX Tau A & 25 & 1 & -8.0 & 4 \\
\hline
\end{tabular}

Notes. ${ }^{\text {a }}$ Estimated from full width of $\mathrm{H} \alpha$ at $10 \%$ level and Equation (1) of Natta et al. (2004).

References. (1) Andrews et al. 2011; (2) Brown et al. 2007; (3) Brown et al. 2009; (4) Espaillat et al. 2010; (5) Fernandez et al. 1995; (6) Garcia Lopez et al. 2006; (7) Hughes et al. 2009; (8) Johns-Krull \& Gafford 2002; (9) Natta et al. 2006; (10) Schisano et al. 2009; (11) White \& Ghez 2001.

up to $75 \mathrm{AU}$, and significant accretion represents a significant subset of transitional disks, and a subset that continues to increase in number (Andrews et al. 2011). In Table 1, we list a sample of nine rapidly accreting transitional disks that have holes between $15 \mathrm{AU}$ and $>70 \mathrm{AU}$ wide.

In general, transitional disks have a variety of observational characteristics and may not represent a single physical phenomenon. For example, clearing driven by the magnetorotational instability (Chiang \& Murray-Clay 2007) can widen dynamically cleared holes once such holes extend all the way to the dust sublimation radius. In Section 3.3, we explain in detail why magnetorotational clearing cannot be responsible for the particular set of transitional disks listed in Table 1. Photoevaporation can also produce disks with inner cleared regions. Prior work on UV photoevaporation showed that evaporation-induced inner holes were inconsistent with accretion rates higher than $\sim 10^{-10} M_{\odot} \mathrm{yr}^{-1}$ (Alexander \& Armitage 2007). In essence, hole formation can only occur once evaporation rates exceed accretion rates, as otherwise the hole will be replenished by accretion. More recently, however, it has been argued that X-ray photoevaporation should be the dominant disk-clearing process and produce much higher mass-loss rates than UV (e.g., Ercolano et al. 2008). Thus, the set of parameter space consistent with photoevaporation has grown to include somewhat higher accretion rates. It is entirely possible that photoevaporation produces some transitional disks, while dynamical clearing by planets produces others. However, even X-ray photoevaporation models do not appear to be able to explain the existence of disks with both large holes $\gtrsim 15-20$ AU and significant accretion rates $\gtrsim 10^{-8.5} M_{\odot} \mathrm{yr}^{-1}$ (Owen et al. 2011).

There is yet one more possible explanation for accreting transitional disks with wide holes-clearing by multiple planets, whose individual gaps could overlap to form a wide hole. Since planets have gap-crossing tidal tails and do not completely truncate the disk, a multiplanet system would allow gas and dust to accrete through the gap. In this work, we demonstrate that the particular set of transitional disks with wide holes and high accretion rates listed in Table 1 presents unambiguous evidence for tidal clearing by multiple planets. While Zhu et al. (2011) found that wide, optically thin gaps in accreting disks could not be produced by planets alone, their conclusions were based on the assumption of axisymmetric gas and dust in the hole. Instead, by taking into account the non-axisymmetric flow patterns produced by planets, we argue not only that multiple planets are consistent with high accretion rates and reduced infrared fluxes, but that they represent the only possible explanation for our sample of transitional disks.

We begin our argument for planetary influence on transitional disks in Section 2, where we demonstrate that planetary systems provide a viable explanation for the gas confinement, non-axisymmetry, and mid-IR flux deficits observed in transitional disks with large holes. In Section 3, we show that the simplest planetary configuration - a single gas giant inside the hole-cannot explain the transitional disks with wide holes. We present dynamical models of multiple planets opening overlapping gaps in Section 4. In Section 5, we use the density distributions predicted by the dynamical model in Section 4 to produce SEDs, and discuss compatibility with observed photometry. In Section 6, we delve into the photoevaporation mechanism in more detail, demonstrating conclusively that it is not a viable originator of our sample of transitional disks. Finally, we present conclusions and testable predictions in Section 7.

\section{PROPERTIES OF TRANSITIONAL DISKS WITH LARGE HOLES}

The primary observational characteristic that defines all transitional disks is that the near- and/or mid-IR fluxes are lower than those observed from "typical" protoplanetary disks. Since the total flux emitted from an optically thick disk is not affected by small changes in the disk surface density, the deficit of flux in transitional disks is generally interpreted as requiring depletion sufficient to make the disk vertically optically thin at the fluxdeficient wavelengths. However, it is important to keep in mind that the actual observable is the emitted flux, not the optical depth, $\tau_{\nu}$. Knowledge of optical depth is required to convert observed fluxes into disk surface densities, but the assumption that $\tau_{v}$ is uniform throughout the hole, or even constant within a given annulus, can lead to serious errors in estimating the mass distribution in the inner disk. In particular, confined, optically thick "streamers" within a hole can produce the same emergent flux as azimuthally symmetric, optically thin dust, while hiding a significant amount of the dust and gas that must move through the hole to match observed accretion rates. If the grains in a narrow tidal tail cover more surface area than the tidal tail itself, so that the tail is locally optically thick, some of the grain mass is effectively hidden. Clumping in Saturn's rings produces exactly this type of plateau in apparent optical depth as a function of particle surface density (Robbins et al. 2010).

In the case of optically thick tidal tails surrounded by empty space, one can simply replace $\tau_{v}$ with a geometrical "filling factor," $f(r)$ - the fraction of an annulus's area covered by tidal tails - in the standard equation for disk flux:

$$
F_{v}=\int_{r_{\text {in }}}^{r_{\text {out }}} 2 \pi r d r B_{v}[T(r)] f(r) / d^{2},
$$

where $F_{\nu}$ is the flux at a given frequency, $r_{\text {in }}$ and $r_{\text {out }}$ are the disk inner and outer radii, respectively, $B_{v}$ is the Planck function in frequency space, $d$ is the distance to the system, and $T$ is the dust temperature at a given radius. An important difference between $f$ and optical depth $\tau_{v}$, however, is that $f$ is purely geometric, and so independent of $v$, while $\tau_{v}$ depends on the opacity $\kappa_{v}$, which is a function of $\nu$. Therefore, the mass distribution and/or temperature structure must be different in the two cases in order to produce the same spectrum. In addition, in a realistic disk, 

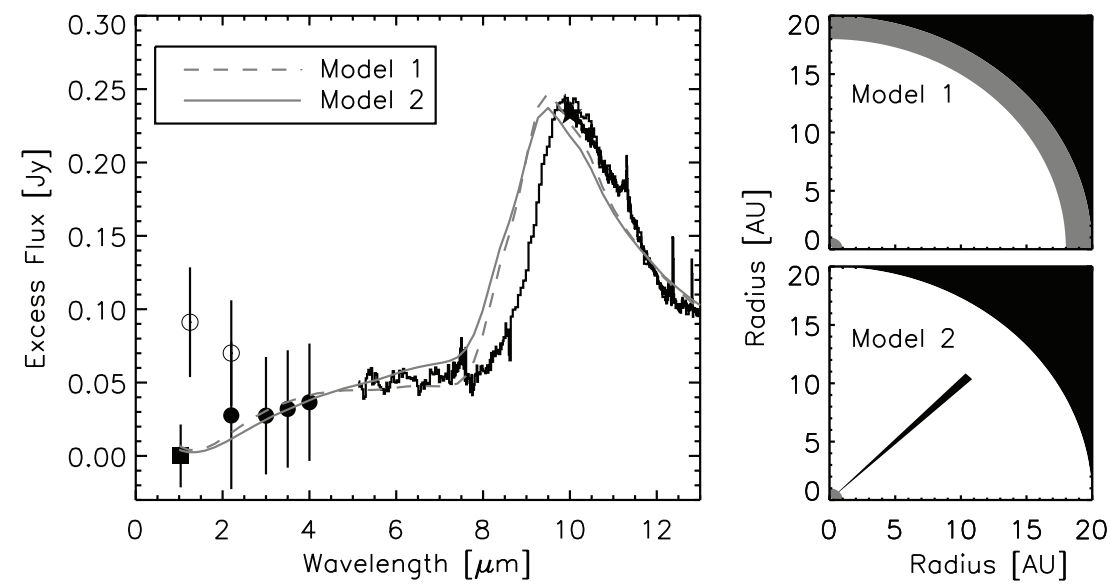

Figure 1. In this figure, we demonstrate some of the degeneracies inherent to modeling of near- and mid-IR excess flux, especially once radial and azimuthal asymmetry are permitted. The left panel shows near- and mid-IR excess fluxes measured for GM Aur with two model SEDs based on different mass distributions (square: Edwards et al. 2006; open circles: Folha \& Emerson 199933; filled circles: Espaillat et al. 20104; star: Weaver \& Jones 1992; spectrum: Calvet et al. 2005; Pontoppidan et al. 2010). ${ }^{5}$ Fluxes are de-reddening using $A_{V}=1.2$ (Espaillat et al. 2010) and the reddening law of Cardelli et al. (1989). The model mass distributions are shown schematically, and to scale, in the right-hand panels, where gray represents optically thin regions, black represents optically thick regions, and white represents empty regions. The outer disk contribution from $R>20 \mathrm{AU}$ is not shown, but primarily contributes at $\lambda>13 \mu \mathrm{m}$.

one must account for the optically thin disk atmosphere, which produces silicate emission features dependent on $\kappa_{\nu}$.

As an example of the ambiguities inherent in modeling transitional disk SEDs, in Figure 1, we show two alternative inner disk dust distributions that produce similar near-IR spectra. To model the resultant flux of the models, we used the radiative transfer code RADMC (similar to Dullemond \& Dominik 2004, but without vertical structure integration) but optically thin components were also benchmarked with an analytical model. The first model consists of an optically thin region from $0.22^{1}$ to $1 \mathrm{AU}$ (with $\Sigma_{\text {dust }}=\Sigma_{0}(R / \mathrm{AU})^{-0.6}$ - used for this and all other models-and $\left.\Sigma_{0}=5 \times 10^{-8} \mathrm{~g} \mathrm{~cm}^{-2}\right)$ and a second optically thin region from 16 to $20 \mathrm{AU}$ (with $\Sigma_{0}=10^{-7} \mathrm{~g} \mathrm{~cm}^{-2}$ ). The second model consists of an optically thin region from 0.22 to $1 \mathrm{AU}$ (with $\Sigma_{0}=3 \times 10^{-8} \mathrm{~g} \mathrm{~cm}^{-2}$ ) and an optically thick streamer from 0.7 to $13 \mathrm{AU}$ (with $f=0.03$ and $H / R=0.08 \times$ $\left.(R / \mathrm{AU})^{1 / 7}\right)$. Both models use the same standard Milky Way dust composition of $80 \%$ small circumstellar silicates and $20 \%$ small carbon grains (Weingartner \& Draine 2001; Li \& Draine 2001; Draine et al. 2007), with silicate opacities from Ossenkopf et al. (1992) and amorphous carbon opacities from Zubko et al. (1996). The carbon grains, being featureless, only act to reduce the line/continuum contrast, and so their quantity is degenerate with other parameters, such as the inner disk small dust grain mass. Optically thin surface densities were chosen to roughly match GM Aur photometric data (although an exhaustive analysis of parameter space was not performed, and clearly these models have some trouble accurately fitting the observed silicate feature). The two models produce nearly identical emergent spectra. Figure 1 demonstrates that significant radial mass transfer can occur through transitional disk holes and still match photometric data, provided the mass is channeled into narrow streams such as planetary tidal tails.

Besides extremely wide inner holes and high accretion rates, many transitional disks contain additional signatures of dynamical perturbation by planetary systems. Brown et al. (2009) noted significant azimuthal asymmetries in the outer disk dust distribution for $\mathrm{LkH} \alpha 330$ and $\mathrm{HD} 135344 \mathrm{~B}$, while Pontoppidan et al. (2008) noted asymmetries in the disks of HD

\footnotetext{
1 The measured inner radius of GM Aur's disk from near-IR interferometric measurements (Akeson et al. 2005)
}

135344 B and SR 21 on the scale of a few AU or less. ${ }^{2}$ Near-IR spectra were suggestive of radial dust asymmetries such as eccentric inner holes in even early modeling studies (e.g., Calvet et al. 2005), and recent studies show that both pre-transitional and transitional disks require multiple inner disk components, including nearly evacuated regions, in order to match observed fluxes and interferometric visibilities (Espaillat et al. 2010; Andrews et al. 2011; Akeson et al. 2011). Similarly, SR 21 has a gas disk truncated at $\sim 7 \mathrm{AU}$ (Pontoppidan et al. 2008), while the dust disk is truncated at $\gtrsim 30 \mathrm{AU}$ (Brown et al. 2009; Andrews et al. 2011). Finally, CO rovibrational line shapes suggest that hot $\mathrm{CO}$ gas in transitional disks may be dynamically confined to smaller regions than in disks without inner holes (Salyk et al. 2011). The tidal barriers of massive planets would provide an extremely effective way to limit the radial distribution of hot gas in transitional disk holes.

A final, unusual characteristic of transitional disks is that they do not seem to arise at any particular star age. Rather, Furlan et al. (2009) show that the transitional disk fraction is $3 \%-4 \%$ in the Taurus, Chamaeleon and Ophiuchus star-forming regions, despite an age spread of 1.5 Myr between the clusters. The transitional disk fractions reported by Currie \& Sicilia-Aguilar (2011) also indicate that disks with inner holes (as opposed to homologously depleted disks) are not preferentially found in clusters of a particular age. The inner hole configuration, then, does not appear to represent an evolutionary stage all disks must go through - in which case there would be a peak in the transitional disk fraction for a given cluster age. Furlan et al. (2009) use their observed transitional disk fractions to argue that the optically thin inner hole configuration lasts about 100,000 years, which is similar to Type II planet migration timescales from the 10 to $15 \mathrm{AU}$ region (Ward 1997). Note, however, the warning by Currie \& Sicilia-Aguilar (2011) that

\footnotetext{
2 Although the asymmetries are observed in the astrometric profile of a $\mathrm{CO}$ emission line, the asymmetry can be present in either the gas or dust component.

3 These veiling values may be high due to a mismatch in photospheric template, or variability (Espaillat et al. 2010).

4 The last three points are estimated from Figure 5 in Espaillat et al. (2010) and are assigned the error bar associated with $r_{K}$.

5 We omit the $M$-band veiling values because of a likely template mismatch in their calculation (Salyk et al. 2009).
} 
there is a degeneracy between the time it takes a process to operate and the frequency with which it operates. It is possible that $10^{5}$ years is the hole-clearing timescale rather than the lifetime of the inner hole.

Planets, therefore, have the potential to provide all the unusual characteristics we observe in the subset of transitional disks under investigation here (Table 1).

1. Wide, apparently optically thin inner holes with $r \gtrsim 15 \mathrm{AU}$.

2. Near and mid-IR flux decrements as compared to classical protoplanetary disks.

3. High accretion rates, up to $10^{-8} M_{\odot} \mathrm{yr}^{-1}$.

4. Confined, non-axisymmetrically distributed gas and dust.

In the next section, we begin by examining the suitability of the simplest planetary configuration, a single gas giant, for opening the wide holes observed in our sample of transitional disks.

\section{ON THE FEASIBILITY OF A SINGLE-PLANET ORIGIN FOR LARGE HOLES}

Clearing by a single giant planet is a commonly invoked explanation for the gaps in transitional disks (e.g., Rice et al. 2003; Espaillat et al. 2008; Furlan et al. 2009). In Section 3.1, we quantify the maximum gap width a planet can open, showing that it is not enough to explain our sample of accreting transitional disks with wide holes. In Sections 3.2 and 3.3, we show that even when one considers the combined effects of a gap-opening planet and accretion or spiral wake-driven clearing of material inside the gap, the timescale for opening a wide hole is outside the inferred $10^{5}$ year transitional disk lifetime/clearing time.

\subsection{Maximum Gap Width}

To build a simple analytical framework for understanding the scope of planetary influence on disks, we begin by imagining the disk as a swarm of massless test particles. Treating the disk as if it were composed of test particles on ballistic trajectories gives the maximum possible gap width a planet can open because gas pressure and viscosity act to fill in the dynamically cleared gap. We estimate the maximum gap width by examining the time it takes for the planet to substantially alter the angular momentum of close-passing particles. The planet opens a gap when repeated encounters cause the particles at a given location to be strongly scattered within the age of the star/disk system. We define strong scattering as a change in the test particle's specific angular momentum of a factor of two to three. The analytical framework used here applies to non-resonant particle orbits.

We consider a test particle with Keplerian frequency $\Omega$ and unperturbed orbital radius $r_{o}$ during a close encounter with the planet, which has Keplerian frequency $\omega$ and orbital radius $r_{p}$. Both the planet and the test particle are initially on circular orbits. In a single encounter, the particle's expected change in specific angular momentum (Lin \& Papaloizou 1993) is

$$
\delta h_{e}=-\frac{64}{243} \frac{G^{2} M_{p}^{2} r_{o}}{\omega^{3} \Delta^{5}}\left[2 K_{0}\left(\frac{2}{3}\right)+K_{1}\left(\frac{2}{3}\right)\right]^{2},
$$

where $M_{p}$ is the mass of the planet, $\Delta=\left|r_{o}-r_{p}\right|$, and $K_{0}$ and $K_{1}$ are modified Bessel functions (Goldreich \& Tremaine 1982). The rate of change in the particle's specific angular momentum due to repeated encounters with the planet is therefore

$$
\dot{h}=\frac{\delta h_{e}}{\delta t},
$$

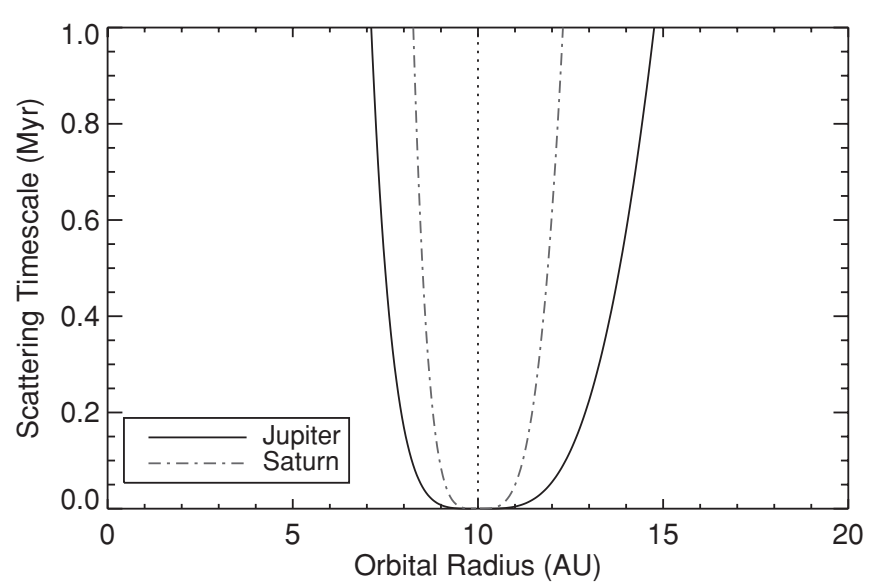

Figure 2. Scattering timescale of particles initially on circular orbits approaches the protostellar disk lifetime for particles more than $\sim 5$ Hill radii from a planet. Here, we plot scattering timescale as a function of distance from the star in a disk containing a planet at $10 \mathrm{AU}$ (vertical dotted line). The high scattering timescales interior to the planet's orbit demonstrate that some other disk dissipation mechanism must act along with planetary clearing to clean out the inner holes of transitional disks.

where the time between encounters is

$$
\delta t=\frac{2 \pi}{|\Omega-\omega|} .
$$

The timescale over which the particle's specific angular momentum changes by of order unity is simply

$$
t=\frac{h}{\dot{h}}=\frac{r_{o}^{2} \Omega}{\dot{h}} .
$$

Figure 2 shows the scattering timescale of disk particles orbiting near two hypothetical planets, one with Jupiter's mass and the other with Saturn's mass. Both planets are placed $10 \mathrm{AU}$ from a Sun-like star. Each planet's tidal radius, or Hill radius, is defined by

$$
R_{H}=r_{p}\left(\frac{M_{p}}{3 M_{*}}\right)^{1 / 3},
$$

given a stellar mass of $M_{*}$. For test particles within three Hill radii of the planet's orbit, the scattering timescale is $10^{4}$ years or less. Tidal interactions with planets are therefore extremely efficient at opening gaps in protoplanetary disks. Notably, Crida et al. (2006) found that even in strongly accreting disks with kinematic viscosity of order $10^{15} \mathrm{~cm}^{2} \mathrm{~s}^{-1}$ at $1 \mathrm{AU}$, corresponding to an accretion rate of $\dot{M}_{*} \approx 5 \times 10^{-7} M_{\odot} \mathrm{yr}^{-1}$ (Dodson-Robinson et al. 2009a), a Jupiter-mass planet (1 $\left.M_{J}\right)$ can open a gap in only 1000 orbits. Encouragingly, planets can open gaps even in disks where strong accretion would readily fill in gaps created by photoevaporative winds.

There is, however, a limited scope to the tidal influence of the planet. As we see from Figure 2, the scattering timescale for particles located four Hill radii or more from the planet approaches the ages of transitional disk host stars. Setting a high scattering timescale limit of $5 \times 10^{5}$ years, which is one-half to one-quarter the estimated stellar age in the Taurus-Auriga association (Palla \& Stahler 2000) and five times higher than the inferred transitional disk lifetime/clearing time (Furlan et al. 2009), we find that the gap opened by a planet may not exceed $\sim 5$ Hill radii. And, since the Hill radius scales as $M_{p}^{1 / 3}$, simply increasing the planet mass does not provide a commensurate 
widening of the gap. Even for a $5 M_{J}$ planet at $10 \mathrm{AU}$, the Hill radius is only $1.2 \mathrm{AU}$, which allows a gap to open from $4 \mathrm{AU}$ to $16 \mathrm{AU}$. This gap is simply not wide enough to account for the observed hole sizes of any of the transitional disks listed in Table 1.

Given the sharp increase in scattering timescale as a function of distance from the planet (Figure 2), our maximum gap width is not highly sensitive to our assumed gap-opening timescale. Our order-of-magnitude estimate of maximum gap width agrees with the numerical work of Crida et al. (2006). Using analytical and numerical calculations of the balance between torques from the planet and viscous stresses, they found that a Jupiter-mass planet in a disk with aspect ratio $H / r=0.05$ opened a gap with a full radius of $5.5 R_{H}$ between the planet orbit and the primordial, unperturbed disk. The width of the optically thin gap is, of course, much smaller than the full center-to-wall width. Our calculation of $5 R_{H}$ as the maximum gap size is actually rather generous.

\subsection{Interior Disk Clearing by Viscous Accretion}

Our calculations demonstrate that a single planet cannot clear a gap wider than $\sim 5$ tidal radii over typical $\mathrm{T}$ Tauri star ages. A Jupiter-mass planet at 10 AU could therefore open a gap extending from 6.5 to 13.5 AU. However, the star is accreting: could the accretion flow drain the disk interior to the planet's orbit (which we hereby label the "interior disk") within the transitional disk lifetime and/or clearing time? For a disk with steady-state mass transfer, the diffusion timescale is

$$
t_{\text {diff }}=\frac{M\left(r_{g}\right)}{\dot{M}_{*}},
$$

where $\dot{M}_{*}$ is the star accretion rate and $M\left(r_{g}\right)$ is the interior disk mass. A derivation of Equation (7) is in Appendix A. For narrow annuli of material located near the star, so that $r_{g} \lesssim 2$ $\mathrm{AU}$, clearing of the interior disk by viscous accretion seems possible. Consider a disk with a surface density profile of

$$
\Sigma(r)=\left(808 \mathrm{~g} \mathrm{~cm}^{-2}\right)\left(\frac{1 \mathrm{AU}}{r}\right)
$$

and an inner edge due to magnetospheric truncation at $0.1 \mathrm{AU}$. This is the pre-clearing density profile of the GM Aur disk constructed by Hughes et al. (2009), who inferred a total disk mass of $0.16 M_{\odot}$ and an outer radius of $300 \mathrm{AU}$ from millimeter imaging. If the star accretes at a rate of $10^{-8} M_{\odot} \mathrm{yr}^{-1}$, the diffusion timescale for material in the inner $2 \mathrm{AU}$ is $10^{5}$ years, similar to transitional disk lifetimes/clearing times (Furlan et al. 2009).

However, what about holes that are 20 AU or larger? Once can place a planet at most $5 R_{H}$ from the desired edge of the hole, or at about $14 \mathrm{AU}$ for a $3 M_{J}$ planet orbiting a Sun-like star. (Since $R_{H}$ scales linearly with the planet's orbital radius, we open the widest gap if we place the planet as far from the star as possible.) The inside edge of the planet's gap is then at about 8 AU. For the inferred GM Aur density profile, the diffusion timescale for material at $8 \mathrm{AU}$ is now $4 \times 10^{5}$ years, edging toward a significant fraction of star ages in the Taurus, Ophiuchus, and Coronet associations (Furlan et al. 2009). Furthermore, the diffusion timescale is really an $e$-folding time (Appendix A), not a time in which complete clearing of interior disk material occurs.

To check how many $e$-foldings it takes to make our interior disk optically thin at millimeter wavelengths, where the wide transitional disk holes have been imaged (Brown et al. 2008; Hughes et al. 2009; Andrews et al. 2011), we use the opacity law

$$
\kappa_{\lambda}=0.02\left(\frac{1 \mathrm{~mm}}{\lambda}\right)^{\beta} \mathrm{cm}^{2} \mathrm{~g}^{-1}
$$

(Beckwith et al. 1990). In Equation (9), $\kappa_{\lambda}$ is the total mass opacity (gas + dust) at a given wavelength $\lambda, \beta$ is an exponent near unity, and gas and micron and/or submicron grains are mixed with a mass ratio of 100:1. Throughout this article we will use the convention that $\kappa_{\lambda}$ refers to an opacity based on the total mass, gas and dust together, and $\kappa_{\lambda \text {,dust }}$ gives an opacity referenced to the mass of dust grains only.

The vertical optical depth of the disk is simply

$$
\tau_{\lambda}=\kappa_{\lambda} \Sigma \text {. }
$$

A surface density of only $20 \mathrm{~g} \mathrm{~cm}^{-2}$ of gas and dust mixed with a 100:1 mass ratio is sufficient to make a disk optically thick, $\tau=1$, at $\lambda=0.4 \mathrm{~mm}$. Diffusing the $100 \mathrm{~g} \mathrm{~cm}^{-2}$ initially at $8 \mathrm{AU}$ in our reconstructed GM Aur disk down to optically thin levels would take two $e$-foldings, or $8 \times 10^{5}$ years. Even for our hypothetical gap with an inner edge at $2 \mathrm{AU}$, for which the characteristic diffusion timescale is seemingly reasonable, three $e$-folding times are required to get to optically thin levels, or $3 \times 10^{5}$ years. If a planet is far enough from its host star for the outer edge of its gap to be at 15-20 AU or more, there is a severe timescale problem with clearing its interior disk to optically thin levels by viscous accretion. As we explore in the next section, the mid-IR limits on leftover surface density from the interior disk are even stronger than the limits from millimeter observations.

\subsection{Interior Disk Clearing by Other Mechanisms}

Interior disk material could be cleared via the scenario proposed by Varnière et al. (2006), in which spiral waves excited by a single planet drive enhanced angular momentum transport that clears the interior disk within $\sim 0.16 t_{\text {diff }}$. Using two-dimensional (2D) hydrodynamic simulations of planet-disk interactions, Varnière et al. show that within 2000 orbits, the planet's spiral wakes reduce the interior disk surface density by about a factor of 10 . To test the viability of spiral wakedriven clearing, we once again set a target hole size of $20 \mathrm{AU}$ to match the GM Aur disk and place a hypothetical planet at $14 \mathrm{AU}, 5 R_{H}$ from the edge of the hole. Our hypothetical planet takes $10^{5}$ years to complete 2000 orbits and open its incomplete hole-so far the clearing time matches the transitional disk lifetime predicted by Furlan et al. (2009).

But an axisymmetric factor of 10 surface density reduction is not nearly enough to make an optically thin hole in the midinfrared. Using a micron/submicron grain opacity of $\kappa_{\lambda \text {,dust }}=$ $500 \mathrm{~cm}^{2} \mathrm{~g}^{-1}$ at $13 \mu \mathrm{m}$ (Ossenkopf et al. 1992), a gas/small grain mass ratio of 100 and Equation (10), we see that the total (gas+dust) surface density required for a disk to be vertically optically thin at $13 \mu \mathrm{m}$ - the spectral index that usually diagnoses flux deficits in transitional disk SEDs (Furlan et al. 2006) - is only $0.2 \mathrm{~g} \mathrm{~cm}^{-2}$. For spiral waves to reduce the surface density from $100 \mathrm{~g} \mathrm{~cm}^{-2}$ to $10 \mathrm{~g} \mathrm{~cm}^{-2}$ at our $8 \mathrm{AU}$ interior disk edge still leaves 50 times too much material to have an optically thin disk at $13 \mu \mathrm{m}$. Single planets and their spiral wakes therefore do not clear enough material from the interior disk to explain observed transitional disk SEDs. However, our submicron grain opacity value is calculated assuming that the grains have maximum sizes of $1 \mu \mathrm{m}$ or less. It is possible 
that the combination of a single planet, wake-driven hole clearing, and locking away $98 \%$ of the solid mass in pebbles, planetesimals, and/or planet cores could be consistent with observed transitional disk fluxes.

We have also considered whether photoevaporation or the magnetorotational instability ("MRI"; Chiang \& Murray-Clay 2007) could clear the interior disk material. As we discuss in detail in Section 6, photoevaporative clearing is only efficient at radii $\gtrsim 3 \mathrm{AU}$. In such a scenario, the interior disk still clears via viscous accretion and is subject to the time constraints we have just discussed. The MRI scenario proposed by Chiang \& Murray-Clay (2007) also cannot clear the interior disk material. If the inner disk is already cleared of most small dust grains, $\mathrm{X}$-rays can ionize material at the edge of the hole and produce inside-out hole widening. The accretion rate is then found to scale roughly as the square of the hole size. Inside-out MRI clearing is therefore not effective at initiating the clearing of material from the interior disk (i.e., at small disk radii).

Transitional disks such as GM Aur and $\mathrm{LkH} \alpha 330$, with inner hole radii of $20 \mathrm{AU}$ and $50 \mathrm{AU}$, respectively (Hughes et al. 2009; Brown et al. 2007), cannot be explained by a single orbiting planet. Even if one takes into account accretion or spiral waveinduced clearing of the material left inside the planet's gap, the inner hole size that can be cleared by a single planet in $10^{5}$ years is limited to a few AU. However, multiple planets orbiting the same star would open adjacent gaps which, combined, could form a large inner hole. In the next section, we use numerical simulations to demonstrate how the "overlapping gap effect" can explain even wide inner holes in accreting disks.

\section{DYNAMICAL CLEARING BY MULTIPLE PLANETS}

Our working picture of a transitional disk is an optically thick outer disk surrounding a wide inner hole that produces little near- and mid-infrared flux. The star must also be able to accrete material through the hole at a high rate, as much as $10^{-8} M_{\odot} \mathrm{yr}^{-1}$. Even though a single planet can only open a narrow gap, a system of multiple planets could produce a wide hole if the planet orbits were close enough together to create overlapping gaps. Because the GM Aur system is one of the best-characterized transitional disks known, we use its overall mass, hole size and accretion rate (Table 1) as guidelines for our study of multiple planets in transitional disks.

We describe our hydrodynamic simulations of hole opening by interacting planets in Section 4.1. Then, in Section 4.2, we show that the non-axisymmetric flow patterns produced by planetary systems are the best possible explanation for the high accretion rates of transitional disk host stars.

\subsection{Hydrodynamic Simulations}

Our planet-disk simulations were conducted using the publicly available version of FARGO, a polar, grid-based 2D hydrodynamic code designed specifically for planet-disk interactions written by Frederic Masset (Masset 2000). Like all polar hydrodynamic codes built on the operator-splitting technique, FARGO uses a sequence of three steps-source step, radial transport step, and azimuthal transport step-to evaluate the transport of any hydrodynamic quantity. The FARGO transport algorithm differs from standard codes in that the azimuthal advection is carried out in a reference frame that rotates at the local Keplerian speed, so that hydrodynamic quantities are advected using the residual velocity, $v_{\phi}-\bar{v}_{\phi}$, instead of the inertial azimuthal velocity. A subsequent step corrects for the azimuthal shifts in the field introduced by subtracting out the Keplerian speed. The advantage of the FARGO transport algorithm is that time steps are not Courant-limited by the short dynamical time at the inner disk boundary. FARGO produces up to a 10 -fold speedup in 2D hydrodynamic calculations over a traditional advection procedure, making simulations that cover a significant fraction of the star age tractable.

In order to test whether a stable multiplanet system could reside inside a transitional disk hole, our simulations include both migration-causing torques from the disk and dynamical interactions between the planets. Using the $20 \mathrm{AU}$ hole size of GM Aur's disk and our single-planet gap size of $5 R_{H}$ as a guide, we set up a disk with three planets located about six mutual Hill radii apart. Chatterjee et al. (2008) defined the mutual Hill radius in planet encounters as

$$
R_{H m}=\left(\frac{M_{1}+M_{2}}{3 M_{*}}\right)^{1 / 3}\left(\frac{a_{1}+a_{2}}{2}\right) .
$$

Tightly packed systems with planet orbits spaced less than $4 R_{H m}$ apart strongly scatter on 1-10 Myr timescales and are not likely to survive intact (Chatterjee et al. 2008; Scharf \& Menou 2009). For planet orbits $6 R_{H m}$ apart, the outer $4 R_{H}$ boundary of the innermost planet, measured in individual Hill radii, coincides with the inner $4 R_{H}$ boundary of the next planet in the system. Our orbital spacing therefore favors the creation of overlapping gaps, which can reach $5 R_{H}$, yet maximizes the likelihood of long-term dynamical stability. To recreate the $20 \mathrm{AU}$ hole in the GM Aur disk, we place three planets, each of three Jupiter masses, at distances of 14.3 AU, 6.3 AU, and 2.7 AU. Our planetary configuration would also provide a reasonable match for the holes of DM Tau or DoAr 44 (19 AU and $30 \mathrm{AU}$, respectively; Andrews et al. 2011).

The disk has a constant aspect ratio of $H / r=0.05$, where $H$ is the pressure scale height. We place the outer boundary at $38 \mathrm{AU}$ and the inner boundary at $0.72 \mathrm{AU}$. Our evenly spaced grid contains 220 radial zones and 384 azimuthal zones. The inner boundary is open so that mass can leave the grid, simulating accretion onto the star. We also allow mass to enter the grid at the outer edge of the disk, mimicking a steady-state mass transfer profile. The initial surface density profile of the disk is the same as in Equation (8) (Section 3). If we extrapolate the surface density profile out to $300 \mathrm{AU}$, the observed size of the GM Aur disk (Hughes et al. 2009), the overall disk mass would be $0.16 M_{\odot}$.

Accretion through the disk is parameterized using the $\alpha$-viscosity, where the turbulent diffusion coefficient is

$$
v=\alpha c_{s} H \text {. }
$$

In Equation (12), $c_{s}$ is the sound speed and $\alpha$ is the efficiency with which gravitational energy is extracted from the shear flow and transformed into turbulence. Our choice of $\alpha$ was motivated by studies of turbulence caused by the magnetorotational instability or MRI, the leading contender for the viscosity source in protostellar disks (Balbus \& Hawley 1991; Balbus et al. 1996). Turner \& Sano (2008) find that $\alpha \leqslant 10^{-4}$ inside the optically thick, nonionized "dead zone" within two pressure scale heights of the disk midplane. We adopt a turbulent efficiency of $\alpha=0.002$, consistent with mass-weighted estimates of local accretion rates with turbulence driven by the MRI (Turner \& Sano 2008; Turner \& Drake 2009). All parameters in the FARGO simulation are summarized in Table 2.

Like Zhu et al. (2011), we allow FARGO to apply only the Type II migration torques. In Type I migration, which occurs 
Table 2

Parameters of the FARGO Simulation

\begin{tabular}{lll}
\hline \hline Parameter & \multicolumn{1}{c}{ Description } & \multicolumn{1}{c}{ Value } \\
\hline Disk setup & & \\
$r_{\text {in }}$ & Inner boundary & $0.72 \mathrm{AU}$ \\
$r_{\text {out }}$ & Outer boundary & $38 \mathrm{AU}$ \\
& Grid zones & 220 radial, 384 azimuthal \\
& Boundary conditions & Open with outer mass source \\
$H / r$ & Aspect ratio & 0.05 \\
$\alpha$ & Viscous efficiency & 0.002 \\
& Planet release time & 12,000 years \\
Planet setup & & \\
$M_{p}$ & Planet masses & $3 M_{J}$ \\
$r_{p}$ & Initial orbit radii & $2.7 \mathrm{AU}, 6.3 \mathrm{AU}, 14.3 \mathrm{AU}$ \\
& Initial planet separation & $6 R_{H m}$ \\
\hline
\end{tabular}

\section{Notes.}

a Open boundaries allow mass to move freely through the grid and accrete onto the star. The outer mass source allows material from beyond $38 \mathrm{AU}$, which we do not model, to move into the grid assuming steady-state accretion.

$\mathrm{b}$ To remove unphysical Type I migration torques from our simulation, we hold the planets on fixed circular orbits for 12,000 years (200 orbits of the outermost planet), then turn on migration and few body interactions.

c All planets were initially placed on circular orbits.

before the planet opens a gap, the disk's outward pressure gradient amplifies the torques from the planet's wake at the outer Lindblad resonances, forcing the planet to move inward at an extremely high rate (Ward 1997; Tanaka et al. 2002). In the limit of low thermal diffusivity, the nonlinear torque caused by the "horseshoe" of gas and dust corotating with the planet saturates and the only net torque on the planet comes from the Lindblad resonances (Ward 1991). Yet a new series of investigations of planet migration in viscous disks with nonzero thermal diffusivity demonstrates that the corotation torque may halt inward migration or even drive outward migration (Paardekooper \& Mellema 2006; Kley \& Crida 2008; Kley et al. 2009; Paardekooper et al. 2010, 2011). Since FARGO does not treat heat or momentum diffusion, the migration rates calculated in the Type I limit-either no gap or an incomplete gap-may not accurately describe the motion of a planet in a physically realistic disk. To prevent improper computation of Type I migration torques, we first follow the gap-opening process for 200 orbits of the outermost planet (12,000 years) with the migration feature of the FARGO code turned off. We then "release" the planets, turning on the migration torques and the $N$-body integrator to track planet-planet interactions.

Unlike Zhu et al. (2011), we do not include accretion onto the planets, which can be parameterized in FARGO by specifying the percentage of mass entering the planet's Hill radius that the planet can accrete, $p$. Over the course of both the initial 12,000 year gap-opening epoch in our simulations and the $>10^{5}$ year length of the hole-opening phase (Furlan et al. 2009), a young gas giant shrinks by at least two orders of magnitude, dramatically decreasing its accretion efficiency. A physically realistic estimate of $p$ would therefore require both an adaptive mesh to model the circumplanetary disk in detail and robust knowledge of the interior structure and evolutionary stage of each planet. Because the $3 M_{J}$ planets we have placed in our model transitional disk should be much smaller than their Hill spheres for most of the simulation, we have assumed that accretion onto the planets clears a negligible amount of disk material. In Appendix B, we review the evolution of a young gas giant's interior structure in more detail and discuss the effects of these changes on the planet growth process.

\subsection{Hole Structure and Appearance}

Figure 3 shows the surface density of gas and dust as a function of time in our simulation. A wide hole created by three overlapping individual gaps opens within about 300 orbits of the outermost planet, or $1.8 \times 10^{4}$ years. The color scale in the topmost row of Figure 3 is designed to mimic submillimeter opacities. Based on the opacity law in Equation (9), disk gas and dust mixed with a 100:1 mass ratio with surface density $\sim 20 \mathrm{~g} \mathrm{~cm}^{-2}$ is optically thick $(\tau>1)$ at $400 \mu \mathrm{m}$. All parts of the disk rendered in white are optically thin in the submillimeter. After 400 orbits of the outermost planet, or $2.4 \times 10^{4}$ years, the optically thin submillimeter hole extends to about 17 AU. At $\lambda=1 \mathrm{~mm}$, the hole extends to about $20 \mathrm{AU}$. The appearance of a wide hole consistent with submillimeter observations is not merely a result of having assumed a low-density disk: our initial surface densities are entirely based on the mass of the GM Aur disk inferred from millimeter observations (Hughes et al. 2009), which is quite high at $0.16 M_{\odot}$. Interestingly, Salyk et al. (2009) note that transitional disks with measurable accretion tend to have higher inferred masses than "classical" disks without holes, which supports planet formation as a likely explanation for observed hole structures (although the low-mass transition disk fraction is not yet well known; Andrews et al. 2011).

Furthermore, all transitional disks have mid-IR flux deficits indicating that their inner holes are optically thin from 10 to $25 \mu \mathrm{m}$. On the bottom row of Figure 3, we stretch the color scale to mimic $13 \mu \mathrm{m}$ optical depth. At $13 \mu \mathrm{m}$, micron and submicron grains produce an opacity of $\kappa_{\lambda \text {,dust }}=500 \mathrm{~cm}^{2} \mathrm{~g}^{-1}$ that is not very sensitive to size distribution as long as the maximum grain size is under $10 \mu \mathrm{m}$ (Ossenkopf et al. 1992). The $\tau=1$ threshold is then at a dust-only surface density of $0.002 \mathrm{~g} \mathrm{~cm}^{-2}$ of micron and submicron grains. Assuming a gas/solid mass ratio of 100 and making the assumption that $100 \%$ of the disk's solids are in micron and submicron grains (as opposed to pebbles, planetesimals, or planet cores), the gas and dust together must have a surface density $\Sigma \lesssim 0.2 \mathrm{~g} \mathrm{~cm}^{-2}$ to be vertically optically thin at $13 \mu \mathrm{m}$. Again, all optically thin regions of the disk in the small-grain approximation are rendered in white. Globally, the inner hole has a filling factor of $f \approx 0.5$ and is overall optically thin.

Where a significant portion of the grains have formed pebbles, planetesimals, or planets, the optically thin threshold surface density of gas and solids mixed with a 100:1 mass ratio is higher than $0.2 \mathrm{~g} \mathrm{~cm}^{-2}$. In Figure 4, we once again render the $400 \mu \mathrm{m}$ and $13 \mu \mathrm{m}$ optical depths calculated from orbits 197 and 395 of our hydrodynamical simulation, accounting for the fact that a significant fraction of the available solid mass must be locked away in giant planet cores, residual planetesimals, or planetesimal precursor particles. Our fiducial opacity of $\kappa_{\lambda \text {,dust }}=500 \mathrm{~cm}^{2} \mathrm{~g}^{-1}$ for dust at $13 \mu \mathrm{m}$ is valid to about $20 \%$ for any single grain size or size distribution as long as the maximum grain size present is of order $1 \mu \mathrm{m}$. Yet Mie scattering calculations by Ossenkopf et al. (1992) show that for the Mathis, Rumpl and Nordsieck (MRN) grain size distribution,

$$
\frac{d \log n(a)}{d \log a}=-3.5,
$$

where $n$ is the number of grains and $a$ is the grain radius (Mathis et al. 1977), significant $13 \mu \mathrm{m}$ opacity reductions begin when the 

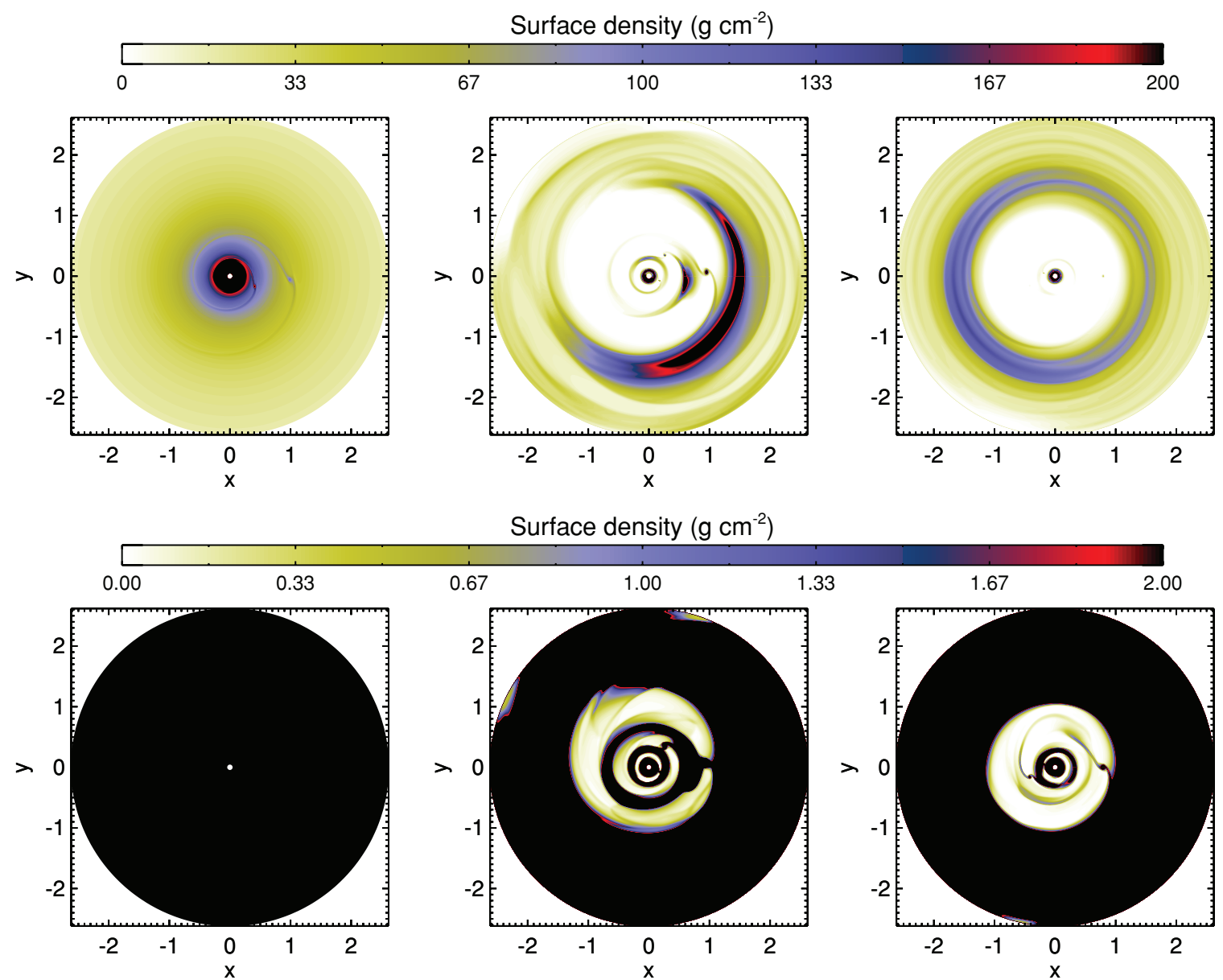

Figure 3. Top row: at $20 \mathrm{AU}$ wide, the hole in the GM Aur disk is too large to have been cleared by a single planet. Three medium-mass planets, all $3 M_{J}$ with distances 14.3 AU, 6.3 AU, and 2.7 AU from GM Aur, clear gaps that overlap and form a large hole over the course of 300 orbits of the outer planet ( $2 \times 10^{4}$ years). Here, we show plots of the disk surface density from orbits 2, 197, and 395 of the FARGO simulation, with the color scale chosen so white regions are optically thin at millimeter wavelengths. The axes show position in the corotating frame, where the outermost planet is at $(x, y)=(1,0)$. Bottom row: here, we demonstrate that tidal streams can efficiently transport mass through a hole that appears to be optically thin in the near- and mid-infrared. Simulation time points are the same as in the top row, but the color scale is designed so that all white regions are optically thin at $13 \mu \mathrm{m}$-assuming gas/solid mass ratio of 100 , all of the disk's solid mass is in micron or submicron grains, and $\kappa_{\lambda \text {,dust }}=500 \mathrm{~cm}^{2} \mathrm{~g}^{-1}$ for the grains (Ossenkopf et al. 1992). Note how the non-axisymmetric, confined flow pattern produced by the planets keeps most of the hole empty while allowing mass transport through locally optically thick tidal streams.

(A color version of this figure is available in the online journal.)

maximum grain size is extended to $10 \mu \mathrm{m}$. Extending the MRN distribution to millimeter, "pebble" sizes produce a factor of 10 opacity reduction over our fiducial value of $\kappa_{\lambda \text {,dust }}$. In Figure 4, we assume that $80 \%$ of solids have either grown to millimeter sizes or become incorporated in planetesimals/planets and the remaining $20 \%$ of the solids are micron and submicron grains which provide the observed flux. The optically thin threshold surface density of gas and solids mixed with a 100:1 mass ratio is then $\Sigma \lesssim 1 \mathrm{~g} \mathrm{~cm}^{-2}$ at $13 \mu \mathrm{m}$ and $\Sigma \lesssim 100 \mathrm{~g} \mathrm{~cm}^{-2}$ at $400 \mu \mathrm{m}$. At $13 \mu \mathrm{m}$, almost $90 \%$ of the inner hole's surface area contains only optically thin dust and the optically thick tidal streams cover only $10 \%$ of the hole, $f \approx 0.1$.

In Figures 3 and 4, we see the detailed structure of each planet's tidal tail, as well as small pileups of material between the planets. Far from being problematic sources of excess flux, these pileups and tails provide a critically important feature of our model: the ability to transport mass through an apparently optically thin hole. The apparent contradiction between optically thin holes and high stellar accretion rates comes from the assumption that gas and grains are evenly and axisymmetrically distributed within the hole (Espaillat et al. 2008, 2010; Zhu et al. 2011). For uniformly distributed grains and a gas/solid mass ratio of 100 , our $0.002 \mathrm{~g} \mathrm{~cm}^{-2}$ of dust, $\tau=1$ threshold would allow less than eight lunar masses of dust in the hole, or $0.1 M_{\oplus}$. If $80 \%$ of the solid mass is in pebbles, planetesimals or planet cores and only the remaining $20 \%$ provides $13 \mu \mathrm{m}$ opacity, the total mass of solids inside the hole could still not exceed $0.5 M_{\oplus}$.

By contrast, after 24,000 years of evolution, our simulated disk's inner hole contains about $0.95 M_{\oplus}$ of solids-yet this material covers between $10 \%$ and $50 \%$ of the surface area of the hole, depending on the assumed gas/small grain mass ratio. In our FARGO simulation, the inner hole contains about Saturn's mass $\left(95 M_{\oplus}\right)$ of gas and dust together. We have therefore used the non-uniform, non-axisymmetric property of flow patterns modulated by giant planets to at least double the mass of small grains consistent with an apparently optically thin inner hole.

Another new piece of observational evidence supports the idea that gas in the inner holes of transitional disks is confined by planets. Using rotation diagrams of the $\mathrm{CO}$ fundamental rovibrational ladder, Salyk et al. (2011) found that simple emission models are consistent with systematically lower surface areas for transitional disks than for gap-free protoplanetary disks. The tidal barriers of planets in the inner holes of transitional disks 


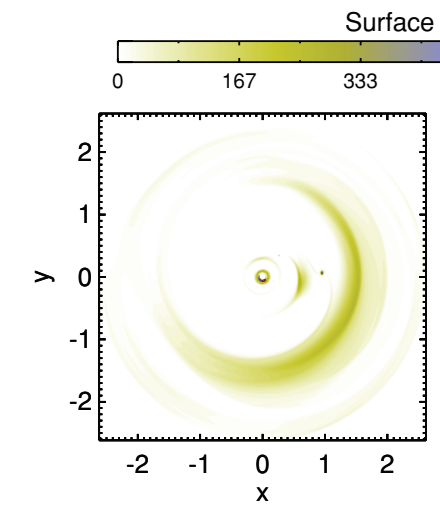

urface density $\left(\mathrm{g} \mathrm{cm}^{-2}\right)$
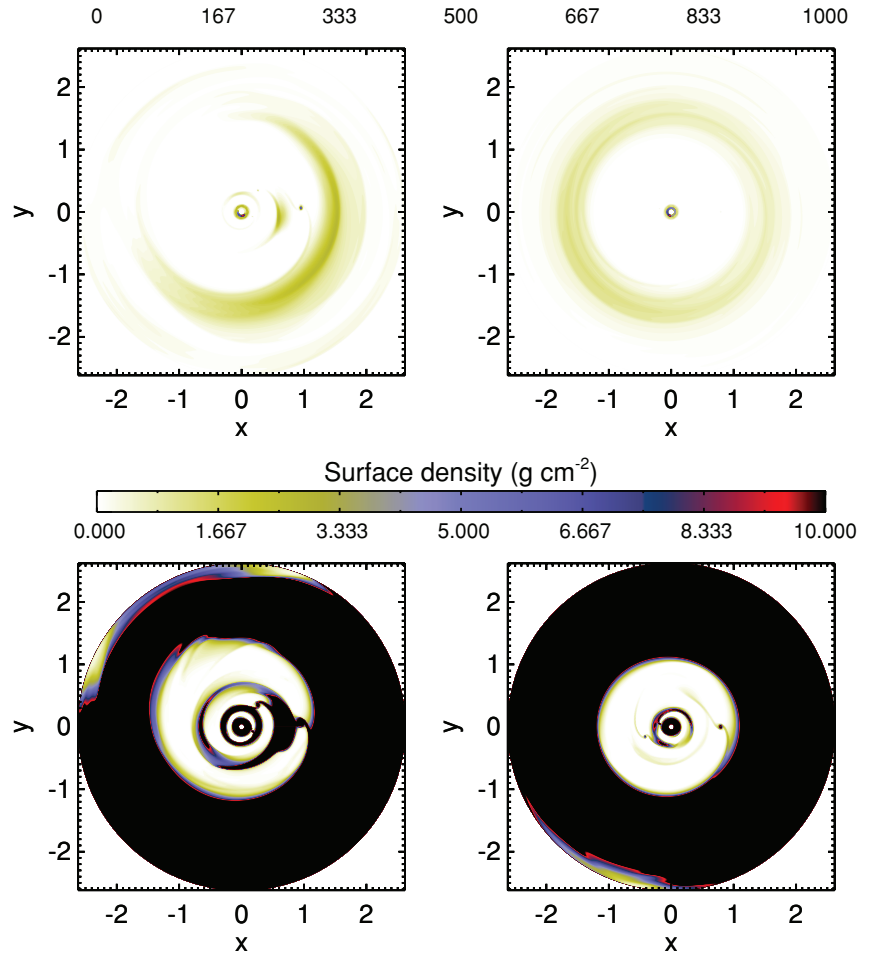

Figure 4. Top row: renderings of the $400 \mu \mathrm{m}$ optical depth of our model disk at orbits 197 and 395 , here assuming that $80 \%$ of the disk's solid mass is in planetesimals and planet cores that do not contribute to millimeter flux. As in Figure 3, all white regions are optically thin. Bottom row: the $13 \mu \mathrm{m}$ optical depth of our model disk if $80 \%$ of the solids have formed pebbles, planetesimals, or planets. After 400 orbits, locally optically thick dust is confined to only about $10 \%$ of the hole surface area, yet the inner hole contains about Saturn's mass in gas and solids. Here, we assume an opacity of $\kappa_{\lambda}=500 \mathrm{~cm}^{2} \mathrm{~g}^{-1}$ for micron/ submicron grains. The total gas/solid mass ratio is still 100.

(A color version of this figure is available in the online journal.)

would confine hot gas to tidal tails or narrow annuli between planets and therefore lead to a lower emitting area for hot gas in transitional disks.

One issue highlighted by Zhu et al. (2011) was their difficulty keeping the planetary system stable. They found that migration drives the planets into 2:1 resonances, which leads to strong scattering. The high migration rate in their simulations is due to their choice of $\alpha$ in the standard $\alpha$-viscosity parameterization: Zhu et al. used $\alpha=0.01$, where we use $\alpha=0.002$, motivated by simulations of MHD turbulence (Section 4.1). Type II migration operates on the disk's viscous timescale, which is shorter for higher values of $\alpha$. The planets in our simulations do begin to migrate after they are released, particularly the outermost planet, which moves from 14.3 AU to $10 \mathrm{AU}$ over the first $5 \times 10^{4}$ years of the simulation. Yet our orbit integration shows that planetary system remains stable for more than $10^{5}$ years. Although physical understanding of turbulent viscosity generation in disks is still limited, it is possible to construct a physically realistic transitional disk in which migration does not destabilize the planetary system.

\section{MODEL TRANSITIONAL DISK SEDs}

In this section, we consider whether the multiplanet dynamical model shown in Figure 3 is consistent with observed photometry of transitional disks. Our goal is to discuss the degree of compatibility between our model and observed fluxes; an exact fit of SED model to data is beyond the scope of this work, and would be hampered by significant limitations. Among the limitations is the fact that the dynamical model predicts only the gas distribution, while most observables are based on emission from small dust grains. Because such grains would be affected by pressure gradients set up by the complex gas structure, and because the expected gas/small dust grain ratio is essentially unknown, the translation from gas distribution to an emission spectrum is not trivial.

Nevertheless, we make some simple assumptions here and produce models designed to give us a qualitative understanding of the compatibility between the multiplanet simulation and transitional disk observations. In particular, we believe that the most stringent requirement is that mid-IR fluxes be as low as observed, as most other features of the disk SED can be fit by tweaking any of the numerous (and degenerate) parameters that enter sophisticated disk models. We begin by comparing our model to photometric data of the GM Aur system. However, we find that our model is in some ways more naturally consistent with pre-transitional disks. We therefore also discuss the pretransitional disk DoAr 44.

Our simple model consists of three components: an optically thin inner disk from $R_{\text {in }}$ to $0.7 \mathrm{AU}$, an optically thick component from 0.7 to $13 \mathrm{AU}$ with a constant filling factor $f$ meant to represent the dynamical model described in Section 4, and an optically thick outer disk from $13 \mathrm{AU}$ to $R_{\text {out }}$ (see Figure 5). Model SEDs are created using the Monte Carlo radiative transfer code RADMC (Dullemond \& Dominik 2004), again without vertical structure integration. Stellar parameters and disk inclination are set to known values (Hughes et al. 2009; Andrews et al. 2011). We use a standard Milky Way dust composition of $20 \%$ carbon and $80 \%$ small (submicrometer) circumstellar silicates (Weingartner \& Draine 2001; Li \& Draine 2001; Draine et al. 2007). $R_{\text {in }}$ was set at the measured dust inner radius, if known, or at roughly the dust sublimation radius, and the mass and radial extent of optically thin dust were adjusted to roughly match observations. To simulate an outer disk that is not directly irradiated by stellar flux but instead mostly shadowed by tidal streams, we took the difference between a model from $R_{\text {in }}$ to $R_{\text {out }}$ and another model from $R_{\text {in }}$ to $13 \mathrm{AU}$-although, as is apparent in Figure 5, the observations do seem to require partial illumination to "puff up" the hole's outer edge (e.g., Andrews et al. 2011; Hughes et al. 2009). However, the flux from the outer disk is not the focus of this work, and we have made no effort to fully explore parameter space for these components.

Instead, we are concerned with the near- and mid-IR flux contribution from the confined tidal streams in our dynamical model. We begin by discussing the mid-IR fluxes, which originate from the $\sim$ few AU region of the disk. The lefthand side of Figure 5 shows the azimuthally averaged dust surface density for our dynamical model after 400 orbits of the outermost planet. If one assumes that the mass is spread evenly over all azimuths, the surface density is almost everywhere optically thick at $13 \mu \mathrm{m}$ (assuming an opacity of $\kappa_{\lambda \text {,dust }}=$ $500 \mathrm{~cm}^{2} \mathrm{~g}^{-1}$ for dust grains). Not surprisingly, since transitional disks by definition have very low $13 \mu \mathrm{m}$ fluxes, an optically thick disk model with $f=1$ (red squares) is not consistent with observations. In reality, however, the density distribution predicted by the dynamical model is not everywhere optically thick, as the mass is shepherded due to the presence of the planets. In the language of Section 2, the azimuthal filling factor, $f(r)$, (gray solid line), ranges from $\sim 0.1$ to 1 if the gas/solid mass ratio is 100 and all solids are in micron and 

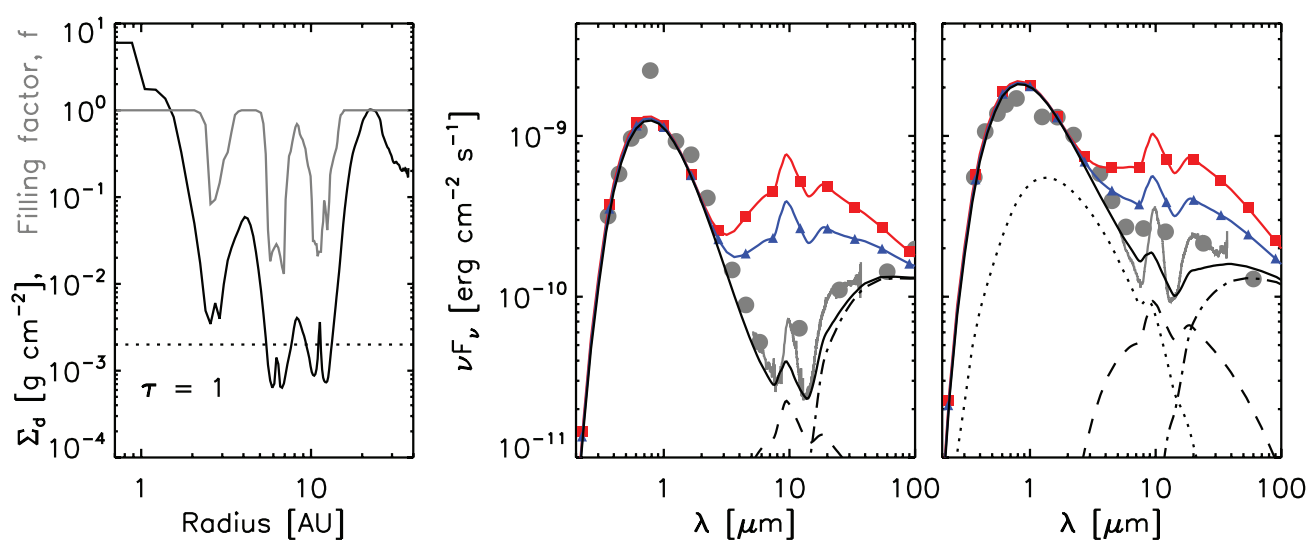

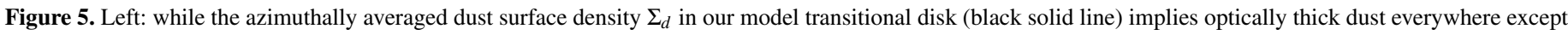

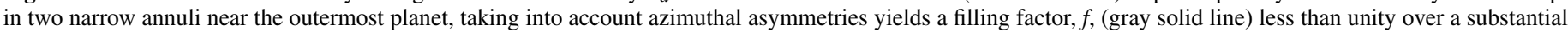

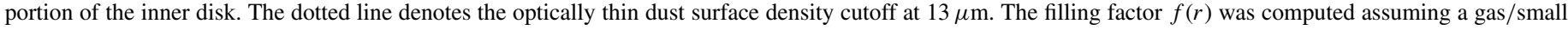

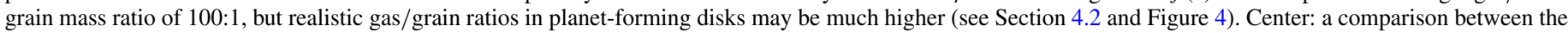

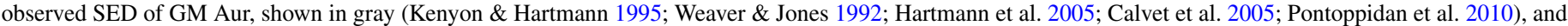

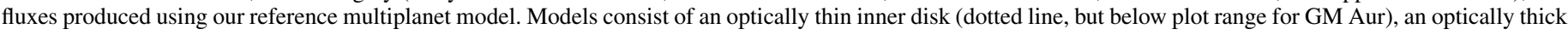

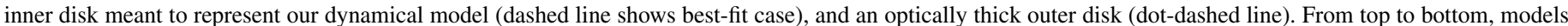

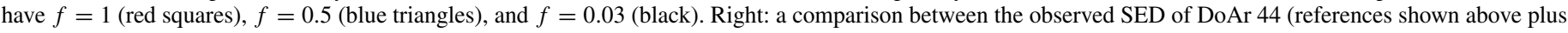
Herbst et al. 1994) and our reference model. From top to bottom, models have $f=1$ (red squares), $f=0.5$ (blue triangles), and $f=0.1$ (black).

(A color version of this figure is available in the online journal.)

submicron grains. Extending the grain size distribution up to millimeter radii or locking away solids in planetesimals and planets correspondingly lowers $f$ (see Figures 3 and 4).

Full 2D modeling of the emergent flux from the distribution predicted by our hydrodynamical simulation is beyond the scope of this analysis; instead, we make the simple assumption that the flux scales proportionally to some characteristic average value of $f$, and ask whether the resulting fluxes are consistent with photometric data. We implicitly assume that optically thin parts of the disk are completely devoid of grains, and so this can be considered a "best-case" scenario. In reality, the optically thin regions would need to have an average $\tau$ lower than that derived from axisymmetric models. On the other hand, we begin with a conservative assumption of the gas/small dust grain ratio, and later discuss the implications of this choice.

The middle panel of Figure 5 compares model SEDs with photometry and IRS spectroscopy of GM Aur. The blue curve (triangles) in Figure 5 shows the flux produced if the inner hole has $f=0.5$, a representative value for our dynamical model if the gas/dust mass ratio is 100 . Although the flux is significantly reduced from the optically thick case, fluxes are still inconsistent with observations. Instead, to match observations, $f$ must be significantly smaller, or $\sim 0.03$ (black curve). For comparison, Calvet et al. (2005) estimate $\tau \sim 0.002$ at $10 \mu \mathrm{m}$. Note that we have not made an effort to fit the silicate feature exactly; however, one can add additional contrast by adding small regions of optically thin dust in the inner disk, and/or by adjusting the grain composition (and thus opacity). Pre-transitional disks like DoAr 44 (right-hand side of Figure 5) are similarly inconsistent with the completely optically thick model (red squares), as well as the $f=0.5$ case (blue triangles), using standard assumptions. However, since the mid-IR fluxes in this case are significantly higher, the required reduction in flux is more moderate-close to $f=0.1$ (black curve).

Are the observations consistent with the dynamical model? As discussed in Section 4.2, $f$ is essentially anti-proportional to the fraction of solid mass locked in pebbles, planetesimals, and planet cores, so one way to match observations of a disk like GM Aur is simply to reduce the mass fraction of small grains in the inner disk, as also suggested by Zhu et al. (2011). That much of the mass has been incorporated into large grains is certainly a reasonable possibility, given the fact that large planets have already formed. It is important to keep in mind that our simple SED models ignore the flux contribution from areas where $\tau<1$ locally, and so the reduction in flux with changes in $f$ due to grain growth is optimistic. Nevertheless, with a powerlaw size distribution of silicate grains with a minimum grain size of $a_{\min }=0.005 \mu \mathrm{m}$, setting $a_{\max }=1 \mathrm{~mm}$ reduces $13 \mu \mathrm{m}$ opacity by a factor of $\sim 10$ relative to a distribution composed entirely of small grains. Therefore, even moderate grain growth can easily make these models a reasonable possibility.

As an alternative to flux reduction by grain growth, one could geometrically constrain the small grains, reducing their filling factor relative to that derived for the gas. It is well known that orbiting dust grains subject to a gas pressure gradient can migrate toward pressure maxima (Weidenschilling 1977), and so it is not unreasonable to expect some additional shepherding of dust grains relative to the gas.

We now consider the near-IR fluxes. One notable feature of our dynamical model (see Figure 3 ) is the persistence of a small annulus of optically thick material between the innermost planet and the star. The dynamical influence of the innermost planet does not extend to these radii, or to radii smaller than $0.7 \mathrm{AU}$, which is the inner boundary of the disk in our dynamical simulation. Thus, the very inner disk is not dynamically cleared, and, if present, contributes significant near-IR flux. As discussed in Section 2, it is possible to hide some optically thick, but very geometrically thin, material in the GM Aur inner disk. However, near-IR fluxes are certainly inconsistent with the large optically thick dust rim extending from 0.2 to $1.5 \mathrm{AU}$ that the dynamical model leaves behind. Therefore, for disks like GM Aur, this inner disk material must be cleared by another mechanism, such as viscous dissipation. Our system of planets was specifically designed to have a viscous clearing timescale for inner disk material of $\sim 10^{5}$ years or less, so this possibility is within reason.

For so-called pre-transitional disks such as DoAr 44, however, near-IR fluxes actually require a significant amount of material 
close to the dust sublimation radius (Espaillat et al. 2010; see also Figure 5). This interior pileup, also found by Rice et al. (2006), is a natural consequence of multiplanet systems of the type described here. In addition, although pre-transitional disks are essentially defined by the presence of this inner disk material, even "traditional" transitional disk sources typically have measurable excess dust emission (e.g., Eisner et al. 2006; Espaillat et al. 2010) as well as emission from hot CO gas (Rettig et al. 2004; Pontoppidan et al. 2008; Salyk et al. 2009) originating at $r<1 \mathrm{AU}$. Thus, our model suggests that the range of observed near-IR fluxes represents a range of stages of dissipation of the material interior to the innermost planet.

\section{OTHER HOLE-OPENING MECHANISMS: PHOTOEVAPORATION}

In Section 4, we showed that systems of multiple planets provide a robust explanation for the observed characteristics of transitional disks: high $\dot{M}$, wide holes that appear optically thin, and confinement of hot gas to small emitting areas. However, another way of opening gaps in disks is to expose the disks to energetic radiation. Here, we discuss the viability of photoevaporation as a hole-opening mechanism. We show that, although photoevaporation can be responsible for transitional disk holes about 3-6 AU wide, it cannot build the extremely wide holes that concern us here.

$\mathrm{X}$-ray, extreme-UV, and far-UV photons transfer kinetic energy to gas in the disk, either directly or through the photoelectric effect, allowing the gas to become unbound from the star. Photoevaporative flows begin at the gravitational radius, where the kinetic energy of the radiatively accelerated gas particle is equal to the escape speed from the star's potential:

$$
R_{g}=\frac{G M_{*}}{c_{s}^{2}} .
$$

In Equation (14), $c_{s}$ is the sound speed in the heated upper layers of the disk. The localization of the photoevaporative flow lends itself well to opening gaps in disks, which may then widen into holes as the interior disk drains on the viscous timescale. However, recall the discussion of the viscous timescale in Section 3: in order for accretion to drain away interior disk material within the lifetime of the disk, the gap must be located within a few AU of the star. Of X-ray, EUV, and FUV photons, only the $\mathrm{X}$-rays have a gravitational radius that might meet our diffusion requirements.

In X-ray photoevaporation, disk clearing begins at a characteristic "clearing age" of 2-4 Myr (Owen et al. 2011). A gap in the disk opens at a characteristic radius of $3 \mathrm{AU}$ and the outer and interior disks are decoupled, so that the interior disk drains on the viscous timescale, several $10^{5}$ years to become optically thin for the surface density profile in Equation (8). X-rays take approximately $10 \%-20 \%$ of the clearing age, or $2 \times 10^{5}$ to $8 \times 10^{5}$ years, to deplete the material between 3 and $10 \mathrm{AU}$. Viscous accretion of material inside $3 \mathrm{AU}$, to leave behind only optically thin dust, and outward expansion of the gap therefore occur on roughly the same timescale. Once the interior disk has been accreted, the disk outside 10 AU is eroded by the $\mathrm{X}$-ray field hitting the hole's inner wall directly on a timescale of the order of the clearing age, a few million years. Accreting transitional disks with inner holes between 3 and $10 \mathrm{AU}$ wide are therefore consistent with the X-ray photoevaporation model. Indeed, Owen et al. (2011) found that approximately $50 \%$ of observed transition disks could be explained by X-ray photoevaporation.
A closer look at the $\mathrm{X}$-ray photoevaporation process, however, reveals why $\mathrm{X}$-ray-driven winds cannot explain our test sample of transitional disks. Once clearing begins to proceed from 10 AU outward via the direct X-ray field, there is no longer any interior mass reservoir to provide accretion onto the star. Accreting disks with large inner holes therefore cannot be explained by X-ray-driven winds. For a $20 \mathrm{AU}$ inner hole, such as in the GM Aur disk, Owen et al. (2011) find a probability of less than $2 \times 10^{-4}$ that the star should still be accreting (see their Figure 12). Furthermore, according to the Owen et al. models, one would have to wait at least 4 Myr to get a 20 AU hole-2 Myr at minimum for the initial gap to open and another $2 \mathrm{Myr}$ for it to move out to $20 \mathrm{AU}$. Their models predict that transitional disks with wide holes should be older than the average disk population, whereas Furlan et al. (2009) and Merín et al. (2010) observe no correlation between transitional disk fraction and cluster age.

We next consider photoevaporation driven by EUV radiation from either the central star or nearby, massive stars. EUV continuum radiation photoionizes the upper layers of a disk so that hydrogen recombination creates a diffuse radiation field that steadily evaporates $\mathrm{H}$ II gas from the disk surface (Hollenbach et al. 1994; Clarke et al. 2001). Once the inner disk has become optically thin to Lyman continuum radiation, the direct UV field also drives a photoevaporative wind that drains the outer disk (Alexander et al. 2006). However, extremeUV photoevaporation only drives disk dissipation when the viscous mass flow through the disk becomes weaker than the photoevaporative flow, estimated by Gorti \& Hollenbach (2009) as

$$
\dot{M}_{\mathrm{EUV}} \lesssim 10^{-9.4} M_{\odot} \mathrm{yr}^{-1}
$$

The stars hosting transitional disks in our test sample have higher accretion rates (Table 1). Models of UV photoevaporation therefore indicate that it cannot be responsible for clearing the holes in our test sample.

Finally, we consider FUV-induced photoevaporation, for which the gravitational radius is outside $30 \mathrm{AU}$ (Matsuyama et al. 2003). Such a large gravitational radius is appealing as a mechanism of opening wide holes, but once more, we have a timescale problem with clearing the disk interior to the gravitational radius. For our reconstructed GM Aur surface density profile (Equation (8)), the single $e$-folding diffusion timescale from $30 \mathrm{AU}$ is $1.7 \mathrm{Myr}$ - higher than the age of GM Aur and other transitional disks in Taurus. Furthermore, because the FUV-driven wind outside the gravitational radius is so much more efficient than viscous accretion inside the gravitational radius, FUV-induced photoevaporation tends to truncate disks rather than opening inner holes (Matsuyama et al. 2003).

Our final piece of evidence that photoevaporation is not responsible for the holes in our sample of transitional disks (Table 1) is its inability to create non-axisymmetric structure: photoevaporative clearing proceeds evenly outward from the gravitational radius. Photoevaporation therefore does not set up the confined accretion flow necessary to move mass efficiently through an optically thin hole. Multiple planets provide the only disk-clearing mechanism that can simultaneously account for the wide inner holes, high accretion rates, confinement of hot gas, and asymmetry observed in transitional disks.

\section{CONCLUSIONS AND TESTABLE PREDICTIONS}

While the initial discoveries of transitional disks with wide holes were based on near- and mid-IR flux deficits (often 
measured by the $13 \mu \mathrm{m} / 30 \mu \mathrm{m}$ flux ratio; Furlan et al. 2006), more detailed observational follow-up of a subset of these disks has revealed a number of unusual characteristics, including:

1. Holes of 15 AU-70 AU or more that are too wide to have been cleared by a single planet (Brown et al. 2007, 2009; Hughes et al. 2009; Espaillat et al. 2010; Andrews et al. 2011).

2. Stellar-mass companions orbiting within $4 \mathrm{AU}$ of the central star ruled out at the $99.7 \%$ confidence level for three of our disks in Table 1 (Pott et al. 2010).

3. Accretion onto the star at rates $10^{-9} M_{\odot} \mathrm{yr}^{-1} \lesssim \dot{M} \lesssim$ $10^{-8} M_{\odot} \mathrm{yr}^{-1}$ (White \& Ghez 2001; Johns-Krull \& Gafford 2002; Andrews et al. 2011).

4. Complex radial mass distributions of dust and gas derived from SED models, millimeter images, IR veiling, and IR interferometry (Espaillat et al. 2010; Andrews et al. 2011; Akeson et al. 2011).

5. Non-axisymmetric structure derived from millimeter images, spectro-astrometry, and sparse aperture masking (Brown et al. 2009; Pontoppidan et al. 2008; Huélamo et al. 2011).

In this article, we have shown that multiple planetary systems are the only phenomena that can explain all of the transitional disk characteristics listed above. The other possible disk-clearing mechanisms-massive single planet, companion star, and photoevaporation-have timescale problems for wide holes and do not provide ways to hide accreting mass in holes that appear optically thin.

The planetary system configuration in the multiplanet model must satisfy three constraints. First, each planet must be a gas giant of $\sim 1 M_{J}$ or more so that it can open a gap. Second, the planets must remain on stable orbits for the lifetime and/or clearing time of the transitional disk lest they disrupt the outer, optically thick disk outside the hole. Finally, for sources with extremely low near-IR fluxes such as GM Aur, the innermost planet must be located within $\sim 2 \mathrm{AU}$ of the central star so that the interior disk pileup can diffuse away within the hole-clearing timescale. For the disks such as DoAr 44 with large holes in millimeter images but higher near-IR fluxes, the constraint on the location of the innermost planet is somewhat relaxed.

Central to our success at finding a single, unifying theory for the wide-hole transitional disks is our break away from assuming axisymmetric, radially uniform dust and gas distributions. In illustrating how accreting mass can be hidden in apparently optically thin holes (Figures 1, 3, and 5), we are using a geometrical definition of optical depth-the macroscopic fraction of an emitting surface area that is covered with light sources. Tidal tails and narrow annuli of dust and gas trapped between planets are locally optically thick, but cover only a small fraction of the inner hole. Overall, then, the inner hole can appear optically thin, but still transport mass onto the star. Our hydrodynamic simulations suggest that taking into account the possibility of a non-uniform dust and gas distribution at least doubles the amount of dust that is consistent with an optically thin inner hole.

The assumption of axisymmetrically distributed gas and dust is problematic because it may hide the dynamical effects of planetary systems. The existence of confined "funnel flows" raises the possibility that time variability of the star accretion rate or excess near-IR flux may be a signature of planetary influence. Accretion rate or near-IR variability would occur on the orbital timescale of the innermost planet. Espaillat et al. (2011) detected variability on 2-3 year timescales in all but 2 of the 14 transitional and pre-transitional disks in their study of the Taurus and Chamaeleon star-forming regions.

The level of axisymmetry in inner disk flow patterns is an observational test of our multiplanet theory of transitional disk origins. As discussed in Section 2, non-axisymmetric structures have been discovered in a number of transitional disks (Brown et al. 2008; Pontoppidan et al. 2008; Huélamo et al. 2011). Embedded protoplanets are also expected to produce asymmetries in emission line profiles (Regály et al. 2010). Furthermore, the finding of Salyk et al. (2011) that hot CO gas in transitional disks has a different, possibly more confined, radial profile as compared to classical disks should be investigated further with disk models. If confirmed, hot gas confinement strongly indicates a dynamical origin for transitional disk holes rather than radiation-driven clearing.

Finally, we offer a possible explanation, based on our multiplanet theory, for the intriguing SR 21 disk which we mentioned in Section 2. SR 21 has a gas disk with a $7 \mathrm{AU}$ inner hole but a dust disk with a 30 AU hole (Pontoppidan et al. 2008; Brown et al. 2009; Andrews et al. 2011). If grain growth and planetesimal formation in SR 21 have proceeded to the point where very few primordial, small grains remain, any observed dust would be second generation, supplied by colliding planetesimals. As we have seen in Section 3.1, giant planets are extremely effective at scattering test particles away from their orbits. A system of gas giants extending to $\sim 30 \mathrm{AU}$ would deplete the region of planetesimals, choking off any chance of producing secondgeneration dust. Gas, however, could still accrete through the planetary system, explaining the smaller gas truncation radius. The SR 21 system fits with our multiplanet theory as long as the observed dust is primarily the product of planetesimal collisions.

As high angular resolution astronomy advances, we urge observers to focus planet-hunting campaigns on transitional disks. ALMA will provide detailed kinematic and spatial information about the gas and dust distributions in transitional disk holes. For the extremely wide, 70 AU holes reported by Andrews et al. (2011), direct imaging with the Gemini Planet Imager may yield pictures of young planets in formation. Finally, aperturemasking techniques have the potential to reveal planetary-mass objects within $\sim 15 \mathrm{AU}$ of their central stars (e.g., Kraus et al. 2009) and may uncover that the hidden planetary systems we suggest are inside the transitional disk holes. In fact, such a companion may have already been detected in the wide transitional disk T Cha (Huélamo et al. 2011). Intriguingly, the only planets that can open gaps in disks are gas giants of the order of Jupiter's mass. Transitional disks have excellent discovery potential for young, still-forming planets, as planet searches focusing on young stars are sensitive only to massive planets.

The discovery of extremely wide holes in transitional disks (Brown et al. 2007; Andrews et al. 2011) points to a possible gravitational-instability origin for the planets contained in the holes: Dodson-Robinson et al. (2009b) demonstrated that gas giants with semimajor axes $r_{p} \gtrsim 35$ AU can only form by gravitational instability. As a bonus, gravitational instability tends to produce hotter, more massive planets than core accretion (Clarke 2009; Stamatellos \& Whitworth 2009), exactly the types of planets best suited to discovery by direct imaging and aperture masking. In contrast, radial velocity observations of main-sequence stars, which have provided almost all of the 500 and more exoplanets discovered to date, are strongly biased toward planets formed by core accretion, which is effective for 
shorter-period orbits. Short-period planets' inability to open wide holes in disks could also explain why radial velocity surveys of young stars with disks, which were designed to include large fractions of transitional disks (e.g., Prato et al. 2008), have yet to detect a hot Jupiter. In any case, studies of transitional disks will likely provide insight into a newly confirmed and only partially understood planet formation mechanism.

The authors thank undergraduate intern Jeffrey Kerbow for his diligent work in Summer 2010, and Joanna Brown, Sean Andrews, and Kevin Covey for insightful discussions about transitional disk observations. We thank Kees Dullemond for making available his radiative transfer code, RADMC, and its supporting documentation, and Frederic Masset for releasing and documenting his 2D hydrodynamic code, FARGO. Support was provided by a University of Texas Dean's Fellowship awarded to S.D.R., the Harlan J. Smith Postdoctoral Fellowship awarded to C.S., and the John W. Cox Endowment for excellence in astronomical research. We also acknowledge computational support from the Texas Advanced Computing Center.

\section{APPENDIX A}

\section{PROTOSTELLAR DISK DIFFUSION TIMESCALE}

To calculate the rate at which disk material accretes onto the star, we consider the surface density diffusion equation (Pringle 1981):

$$
\frac{\partial \Sigma}{\partial t}=\frac{3}{r} \frac{\partial}{\partial r}\left[r^{1 / 2} \frac{\partial}{\partial r}\left(\Sigma v r^{1 / 2}\right)\right],
$$

in which $\Sigma$ is the surface density of disk material, $r$ is the distance from the star, $t$ is time, and $v$ is the kinematic viscosity of the disk material. Over a small region of the disk, one can approximate the diffusion rate by assuming a constant viscosity so that the diffusion equation can be separated into a time component and a space component:

$$
\Sigma(r, t)=R(r) T(t) .
$$

Masset (2000) and Masset \& Papaloizou (2003) also used disks with constant kinematic viscosity in their analytical and numerical studies of planet migration. By taking the partial derivatives of the separated function $\Sigma(r, t)$, we rewrite the diffusion equation as

$$
\frac{1}{v} \frac{T^{\prime}}{T}=\frac{9}{2 r} \frac{R^{\prime}}{R}+3 \frac{R^{\prime \prime}}{R}=-\lambda,
$$

where $T^{\prime}$ and $R^{\prime}$ are partial derivatives over $t$ and $r$, respectively, and $\lambda$ is the constant eigenvalue. The solution to the timedependent portion of Equation (A3) is

$$
T(t)=\exp (-\lambda \nu t)
$$

To find an expression for $\lambda$ that is benchmarked against measured $\mathrm{T}$ Tauri star accretion rates and does not depend on assumptions about viscosity, we examine the mass flow through the interior disk. For an interior disk annulus of finite size such as the one left behind by a gap-clearing planet, one may approximate the total mass transfer through the annulus, $\dot{M}$, as

$$
-\dot{M} \approx \pi r_{g}^{2} \dot{\Sigma}\left(r_{g}\right)
$$

where $r_{g}$ is the inner edge of the gap created by the planet. The negative sign reflects the convention that material flowing inward creates a positive accretion rate onto the star. Substituting $\dot{\Sigma}(r)=R(r) T^{\prime}(t)$ into Equation (A5) and solving for $\lambda$, we find

$$
\lambda \approx \frac{\dot{M}}{\pi r_{g}^{2} \nu \bar{\Sigma}}
$$

where $\bar{\Sigma}$ is the average surface density in the diffusing region $r<r_{g}$.

Using Equation (A6), we may write the solution to the diffusion equation under the assumption of constant viscosity as

$$
\Sigma(r, t) \approx \Sigma(r, 0) \exp \left(\frac{-\dot{M}_{0} t}{\pi r_{g}^{2} \bar{\Sigma}}\right),
$$

where $\dot{M}_{0}$ is the accretion rate at $t=0$. Here, the diffusion timescale we recover is similar to the canonical value $t_{\text {diff }}=$ $r^{2} / v$ (Lynden-Bell \& Pringle 1974), but in terms of the accretion rate:

$$
t_{\text {diff }}=\frac{\pi r_{g}^{2} \bar{\Sigma}}{\dot{M}_{0}}
$$

The quantity $\pi r_{g}^{2} \bar{\Sigma}$ is, of course, equivalent to the disk mass $M\left(r_{g}\right)$ contained inside a circle of radius $r_{g}$, so we rewrite Equation (A8) to get Equation (7) in Section 3.

A standard $\alpha$-viscosity disk model with $\Sigma \propto r^{-1}$, as is usually inferred from submillimeter observations, has a viscosity profile $v \propto r$ so that the radial dependence of $t_{\text {diff }}$ according to the traditional $r^{2} / v$ criterion is linear. Even though we have, for the purpose of clarity, based our derivation of Equation (7) on a disk with constant viscosity, we still recover a linear dependence of $t_{\text {diff }} M\left(r_{g}\right) / \dot{M}_{*}$ on radius. Our diffusion timescale equation applies to a range of commonly used disk models.

\section{APPENDIX B}

\section{ACCRETION EFFICIENCY AND PLANETARY INTERIOR STRUCTURE}

Bodenheimer (1974) divides the evolution of a young gas giant into four phases. The first is a transient hydrodynamic epoch, in which gas at the planet's Hill radius free falls toward the center of the planet: the planet's rapid growth is driven by the runaway expansion of its Hill sphere. The hydrodynamic epoch lasts about one free-fall time, $\sim 1000$ years. For planets growing by core accretion, Phase I encompasses the mass range of $\sim 10-500 M_{\oplus}$. For planets formed by disk fragmentation, the characteristic Phase I mass is much higher (e.g., Clarke 2009). Planets in Phase I accrete $100 \%$ of the material inside their Hill spheres. Gap opening begins during Phase I at about Saturn's mass, $95 M_{\oplus}$, and eventually halts the runaway growth of the planet, which is why we do not start our simulations with small planet cores and let them accrete: FARGO's accretion efficiency parameter $p$ would suffer a dramatic drop during only the first 1000 years of disk evolution.

At the onset of Phase II, the young planet develops an interior pressure gradient that halts the free fall of material at the Hill radius. The planet then stays in Phase II, quasi-static contraction on the Kelvin-Helmholz timescale over about a factor of 20 in radius, for $\sim 10^{5}$ years. The planet's evolution during this period is similar to that of a protostar on the Hayashi track. Accretion during Phase II is limited by the disk's ability to deliver gas to the edge of the gap and by the strength of the planet's tidal barrier (e.g., Dobbs-Dixon et al. 2008). "Leakage" of gas across 
the tidal barrier is much more efficient at the beginning of Phase II, when the planet nearly fills its Hill sphere: at this stage, material from the disk can fall directly to the planet's surface. As the planet contracts, the velocity of disk material entering the Hill sphere increases relative to the planetary surface, so that accreting gas will orbit the planet in a circumplanetary disk (Papaloizou \& Nelson 2005). Mass transfer onto the planet is then regulated by the diffusion timescale in the circumplanetary disk as well as the mass transfer rate through the protostellar disk and is extremely inefficient (Ward \& Canup 2010).

Once the planet's interior reaches $2000 \mathrm{~K}$, molecular hydrogen begins to dissociate, draining the thermal energy that maintains the quasi-static pressure gradient. The resulting supersonic collapse, which starts Phase III, takes only a few days and decreases the planet's radius by a factor of more than 100 , further limiting the planet's ability to accrete. We see that a young planet's accretion efficiency changes dramatically as it evolves. During most of the transitional disk epoch, the planet will be accreting through a circumplanetary disk whose mass transfer efficiency depends on the ratio of protostellar/circumplanetary disk viscosities and the size of the planet. The circumplanetary disk viscosity depends in turn on the density and temperature of circumplanetary material.

Since FARGO is an isothermal code that does not treat the planetary interior structure, it much better suited for parameter studies of gap-opening efficiency in the presence of planetary accretion than physically realistic computations of planet growth profiles. Because the accretion-limiting circumplanetary disk should be present and growing during most of the hole-clearing epoch, we are justified in assuming that mass transfer onto the planets is negligible throughout our FARGO simulation.

\section{REFERENCES}

Akeson, R. L., Boden, A. F., Monnier, J. D., et al. 2005, ApJ, 637, 1173 Akeson, R. L., Millan-Gabet, R., Ciardi, D. R., et al. 2011, ApJ, 728, 96 Alexander, R. D., \& Armitage, P. J. 2007, MNRAS, 375, 500

Alexander, R. D., Clarke, C. J., \& Pringle, J. E. 2006, MNRAS, 369, 216

Andrews, S., Wilner, D., Espaillat, C., et al. 2011, ApJ, 732, 42

Andrews, S. M., Wilner, D. J., Hughes, A. M., Qi, C., \& Dullemond, C. P. 2010, ApJ, 723, 1241

Balbus, S. A., \& Hawley, J. F. 1991, ApJ, 376, 214

Balbus, S. A., Hawley, J. F., \& Stone, J. M. 1996, ApJ, 467, 76

Beckwith, S. V. W., Sargent, A. I., Chini, R. S., \& Guesten, R. 1990, AJ, 99, 924

Bodenheimer, P. 1974, Icarus, 23, 319

Brown, J. M., Blake, G. A., Dullemond, C. P., et al. 2007, ApJ, 664, L107

Brown, J. M., Blake, G. A., Qi, C., Dullemond, C. P., \& Wilner, D. J. 2008, ApJ, 675, L109

Brown, J. M., Blake, G. A., Qi, C., et al. 2009, ApJ, 704, 496

Calvet, N., D'Alessio, P., Hartmann, L., et al. 2002, ApJ, 568, 1008

Calvet, N., D'Alessio, P., Watson, D. M., et al. 2005, ApJ, 630, L185

Cardelli, J. A., Clayton, G. C., \& Mathis, J. S. 1989, ApJ, 345, 245

Chatterjee, S., Ford, E. B., Matsumura, S., \& Rasio, F. A. 2008, ApJ, 686, 580

Chiang, E., \& Murray-Clay, R. 2007, Nature Phys., 3, 604

Clarke, C. J. 2009, MNRAS, 396, 1066

Clarke, C. J., Gendrin, A., \& Sotomayor, M. 2001, MNRAS, 328, 485

Crida, A., Morbidelli, A., \& Masset, F. 2006, Icarus, 181, 587

Currie, T., \& Sicilia-Aguilar, A. 2011, ApJ, 732, 24

Dobbs-Dixon, I., Li, S., \& Lin, D. N. C. 2008, in IAU Symp. 249, Exoplanets: Detection, Formation and Dynamics, ed. Y.-S. Sun, S. Ferraz-Mello, \& J.-L. Zhou (Cambridge: Cambridge Univ. Press), 263

Dodson-Robinson, S. E., Veras, D., Ford, E. B., \& Beichman, C. A. 2009a, ApJ, 707, 79

Dodson-Robinson, S. E., Willacy, K., Bodenheimer, P., Turner, N. J., \& Beichman, C. A. 2009b, Icarus, 200, 672

Draine, B. T., Dale, D. A., Bendo, G., et al. 2007, ApJ, 663, 866

Dullemond, C. P., \& Dominik, C. 2004, A\&A, 417, 159

Edwards, S., Fischer, W., Hillenbrand, L., \& Kwan, J. 2006, ApJ, 646, 319

Eisner, J. A., Chiang, E. I., \& Hillenbrand, L. A. 2006, ApJ, 637, L133
Ercolano, B., Drake, J. J., Raymond, J. C., \& Clarke, C. C. 2008, ApJ, 688, 398 Espaillat, C., Calvet, N., Luhman, K. L., Muzerolle, J., \& D’Alessio, P. 2008, ApJ, 682, L125

Espaillat, C., D’Alessio, P., Hernández, J., et al. 2010, ApJ, 717, 441

Espaillat, C., Furlan, E., D’Alessio, P., et al. 2011, ApJ, 728, 49

Fernandez, M., Ortiz, E., Eiroa, C., \& Miranda, L. F. 1995, A\&AS, 114, 439

Folha, D. F. M., \& Emerson, J. P. 1999, A\&A, 352, 517

Furlan, E., Hartmann, L., Calvet, N., et al. 2006, ApJS, 165, 568

Furlan, E., Watson, D. M., McClure, M. K., et al. 2009, ApJ, 703, 1964

Garcia Lopez, R., Natta, A., Testi, L., \& Habart, E. 2006, A\&A, 459, 837

Goldreich, P., \& Tremaine, S. 1982, ARA\&A, 20, 249

Gorti, U., \& Hollenbach, D. 2009, ApJ, 690, 1539

Hartmann, L., Megeath, S. T., Allen, L., et al. 2005, ApJ, 629, 881

Hatzes, A. P., Cochran, W. D., McArthur, B., et al. 2000, ApJ, 544, L145

Herbst, W., Herbst, D. K., Grossman, E. J., \& Weinstein, D. 1994, AJ, 108, 1906

Hollenbach, D., Johnstone, D., Lizano, S., \& Shu, F. 1994, ApJ, 428, 654

Huélamo, N., Lacour, S., Tuthill, P., et al. 2011, A\&A, 528, L7

Hughes, A. M., Andrews, S. M., Espaillat, C., et al. 2009, ApJ, 698, 131

Ireland, M. J., \& Kraus, A. L. 2008, ApJ, 678, L59

Johns-Krull, C. M., \& Gafford, A. D. 2002, ApJ, 573, 685

Kalas, P., Graham, J. R., Chiang, E., et al. 2008, Science, 322, 1345

Kenyon, S. J., \& Hartmann, L. 1995, ApJS, 101, 117

Kley, W., Bitsch, B., \& Klahr, H. 2009, A\&A, 506, 971

Kley, W., \& Crida, A. 2008, A\&A, 487, 9

Koerner, D. W., Sargent, A. I., \& Beckwith, S. V. W. 1993, Icarus, 106, 2

Kraus, A. L., Ireland, M. J., Martinache, F., \& Hillenbrand, L. A. 2011, ApJ, 731, 8

Kraus, A. L., Ireland, M. J., Martinache, F., \& Lloyd, J. P. 2009, in AIP Conf. Ser. 1094, Cool Stars, Stellar Systems and The Sun, ed. E. Stempels (Melville, NY: AIP), 453

Lagrange, A.-M., Bonnefoy, M., Chauvin, G., et al. 2010, Science, 329, L57

Li, A., \& Draine, B. T. 2001, ApJ, 554, 778

Lin, D. N. C., \& Papaloizou, J. C. B. 1993, Protostars and Planets III, ed. E. Levy \& J. Lunine (Tucson, AZ: Univ. Arizona Press), 749

Lynden-Bell, D., \& Pringle, J. E. 1974, MNRAS, 168, 603

Marois, C., Macintosh, B., Barman, T., et al. 2008, Science, 322, 1348

Marois, C., Zuckerman, B., Konopacky, Q. M., Macintosh, B., \& Barman, T. 2010, Nature, 468, 1080

Masset, F. 2000, A\&AS, 141, 165

Masset, F. S., \& Papaloizou, J. C. B. 2003, ApJ, 588, 494

Mathis, J. S., Rumpl, W., \& Nordsieck, K. H. 1977, ApJ, 217, 425

Matsuyama, I., Johnstone, D., \& Hartmann, L. 2003, ApJ, 582, 893

Merín, B., Brown, J. M., Oliveira, I., et al. 2010, ApJ, 718, 1200

Muzerolle, J., Allen, L. E., Megeath, S. T., Hernández, J., \& Gutermuth, R. A. 2010, ApJ, 708, 1107

Natta, A., Testi, L., Muzerolle, J., et al. 2004, A\&A, 424, 603

Natta, A., Testi, L., \& Randich, S. 2006, A\&A, 452, 245

Ossenkopf, V., Henning, T., \& Mathis, J. S. 1992, A\&A, 261, 567

Owen, J. E., Ercolano, B., \& Clarke, C. J. 2011, MNRAS, 412, 13

Paardekooper, S.-J., Baruteau, C., Crida, A., \& Kley, W. 2010, MNRAS, 401, 1950

Paardekooper, S.-J., Baruteau, C., \& Kley, W. 2011, MNRAS, 410, 293

Paardekooper, S.-J., \& Mellema, G. 2006, A\&A, 450, 1203

Palla, F., \& Stahler, S. W. 2000, ApJ, 540, 255

Papaloizou, J. C. B., \& Nelson, R. P. 2005, A\&A, 433, 247

Pontoppidan, K. M., Blake, G. A., van Dishoeck, E. F., et al. 2008, ApJ, 684 1323

Pontoppidan, K. M., Salyk, C., Blake, G. A., et al. 2010, ApJ, 720, 887

Pott, J.-U., Perrin, M. D., Furlan, E., et al. 2010, ApJ, 710, 265

Prato, L., Huerta, M., Johns-Krull, C. M., et al. 2008, ApJ, 687, L103

Pringle, J. E. 1981, ARA\&A, 19, 137

Quillen, A. C., Blackman, E. G., Frank, A., \& Varnière, P. 2004, ApJ, 612, L137

Regály, Zs., Sándor, Zs., Dullemond, C. P., \& van Boekel, R. 2010, A\&A, 523, 69

Rettig, T. W., Haywood, J., Simon, T., Brittain, S. D., \& Gibb, E. 2004, ApJ, 616, L163

Rice, W. K. M., Armitage, P. J., Wood, K., \& Lodato, G. 2006, MNRAS, 373, 1619

Rice, W. K. M., Wood, K., Armitage, P. J., Whitney, B. A., \& Bjorkman, J. E. 2003, MNRAS, 342, 79

Robbins, S. J., Stewart, G. R., Lewis, M. C., Colwell, J. E., \& Sremčević, M. 2010, Icarus, 206, 431

Salyk, C., Blake, G. A., Boogert, A. C. A., \& Brown, J. M. 2009, ApJ, 699, 330

Salyk, C., et al. 2011, ApJ, submitted

Scharf, C., \& Menou, K. 2009, ApJ, 693, 113

Schisano, E., Covino, E., Alcalá, J. M., et al. 2009, A\&A, 501, 1013

Stamatellos, D., \& Whitworth, A. P. 2009, MNRAS, 392, 413 
Tanaka, H., Takeuchi, T., \& Ward, W. 2002, ApJ, 565, 1257

Turner, N. J., \& Drake, J. F. 2009, ApJ, 703, 2152

Turner, N. J., \& Sano, T. 2008, ApJ, 679, 131

Varnière, P., Blackman, E. G., Frank, A., \& Quillen, A. C. 2006, ApJ, 640, 1110

Ward, W. R. 1991, Lunar and Planetary Science Institute Science Conference Abstracts (Houston, TX: Lunar and Planetary), Vol. 22, 1463

Ward, W. R. 1997, Icarus, 126, 261

Ward, W. R., \& Canup, R. M. 2010, AJ, 140, 1168
Weaver, W. B., \& Jones, G. 1992, ApJS, 78, 239

Weidenschilling, S. J. 1977, MNRAS, 180, 57

Weingartner, J. C., \& Draine, B. T. 2001, ApJ, 548, 296

White, R. J., \& Ghez, A. M. 2001, ApJ, 556, 265

Zhu, Z., Nelson, R. P., Hartmann, L., Espaillat, C., \& Calvet, N. 2011, ApJ, 729, 47

Zubko, V. G., Mennella, V., Colangeli, L., \& Bussoletti, E. 1996, MNRAS, 282, 1321 